NBER WORKING PAPER SERIES

\title{
REGIME-SWITCHING AND THE ESTIMATION OF MULTIFRACTAL PROCESSES
}

\author{
Laurent Calvet \\ Adlai Fisher \\ Working Paper 9839 \\ http://www.nber.org/papers/w9839
}

\author{
NATIONAL BUREAU OF ECONOMIC RESEARCH \\ 1050 Massachusetts Avenue \\ Cambridge, MA 02138 \\ July 2003
}

We are grateful to John Campbell, Guido Kuersteiner and Nour Meddahi for stimulating discussions. We also received helpful comments from T. Andersen, T. Bollerslev, F. Diebold, R. Engle, J. Hamilton, J. MacKinnon, N. Shephard, D. Smith, R. Tsay and seminar participants at the University of British Columbia, the University of Chicago, the University of Pennsylvania, the 2002 NSF-NBER Time Conference, the 2002 CIRANO Extreme Events in Finance Conference, and the 2003 North-American Summer Meeting of the Econometric Society. Excellent research assistance was provided by Xifeng Diao. We are very appreciative of financial support provided for this project by the Social Sciences and Humanities Research Council of Canada under grant 4102002-0641. The views expressed herein are those of the authors and not necessarily those of the National Bureau of Economic Research

(C)2003 by Laurent Calvet and Adlai Fisher. All rights reserved. Short sections of text not to exceed two paragraphs, may be quoted without explicit permission provided that full credit including (C) notice, is given to the source. 
Regime-Switching and the Estimation of Multifractal Processes

Laurent Calvet and Adlai Fisher

NBER Working Paper No. 9839

July 2003

JEL No. G0, C5

\section{$\underline{\text { ABSTRACT }}$}

We propose a discrete-time stochastic volatility model in which regime switching serves three purposes.

First, changes in regimes capture low frequency variations, which is their traditional role. Second, they specify intermediate frequency dynamics that are usually assigned to smooth autoregressive processes. Finally, high frequency switches generate substantial outliers. Thus, a single mechanism captures three important features of the data that are typically addressed as distinct phenomena in the literature. Maximum likelihood estimation is developed and shown to perform well in finite sample. We estimate on exchange rate data a version of the process with four parameters and more than a thousand states. The estimated model compares favorably to earlier specifications both in- and out-of-sample. Multifractal forecasts slightly improve on $\operatorname{GARCH}(1,1)$ at daily and weekly intervals, and provide considerable gains in accuracy at horizons of 10 to 50 days.

Laurent Calvet

Department of Economics

Harvard University

Littauer Center

Cambridge, MA 02138

and NBER

lcalvet@aya.yale.edu
Adlai Fisher

Finance Division

Sauder School of Business

University of British Columbia

2053 Main Mall

Vancouver, BC

Canada V6T $1 Z 2$

adlai.fisher@commerce.ubc.ca 


\section{Introduction}

Over the past fifteen years, stochastic regime-switching (Hamilton 1989, 1990) has proven to be extremely useful for modeling economic and financial time series. ${ }^{1}$ While the theoretical formulation is very general, empirical researchers most commonly apply this approach to low frequency variations and rely on other techniques for shorter-run dynamics. For example, Markov-switching ARCH and GARCH processes separately specify regime shifts at low frequencies, smooth autoregressive volatility transitions at mid-range frequencies, and a thick-tailed conditional distribution of returns at high-frequency (Cai, 1994; Hamilton and Susmel, 1994; Gray, 1996; Klaassen, 2002). In this paper, we propose an alternative volatility model based on pure regime-switching at all frequencies.

Previous empirical applications typically employ only a small number of discrete states. This partly stems from the common view that regime switches occur infrequently. A more practical limitation is that the number of parameters grows quadratically with the cardinality of the state space in a general formulation. Restrictions on state parameters and switching probabilities offer a natural solution, as pursued for example by Bollen, Gray, and Whaley (2000) in a fourregime model. ${ }^{2}$ We extend this approach by considering a tight set of restrictions inspired by the multifractal literature. This permits the routine estimation of good-performing models with over a thousand states, a dense transition matrix, and only four parameters.

Our specification is particularly influenced by an innovation in multifractal

\footnotetext{
${ }^{1}$ The likelihood-based estimation of Markov-switching processes was developed by Lindgren (1978) and Baum et al. (1980) in the statistics literature. A seminal series of papers by Hamilton $(1989,1990)$ introduced these processes to econometrics and spurred the development of a large body of research. Contributions to the original version of the model advance estimation and testing (Albert and Chibb, 1993; Garcia, 1998; Hansen, 1992; Shephard, 1994), and investigate a wide range of empirical applications (e.g. Hamilton, 1988; Garcia and Perron, 1996). The approach has been extended to incorporate GARCH transitions (Cai, 1994; Gray, 1996; Hamilton and Susmel, 1994; Kim, 1994; Kim and Nelson, 1999; Klaassen, 2002), vector processes (Hamilton and Lin, 1996; Hamilton and Perez-Quiros, 1996), and time-varying transition probabilities (Diebold, Lee, and Weinbach, 1994; Durland and McCurdy, 1994; Filardo, 1994; Perez-Quiros and Timmerman, 2000). See Hamilton and Raj (2002) for a recent survey.

${ }^{2}$ Duration-dependent Markov-switching models also use restrictions on state parameters and switching probabilities. For example, Maheu and McCurdy (2000) expand a two-state model by conditioning the volatility level and switching probability on duration in the state. The resulting transition matrix is sparse, and the system either progresses to the next duration of the same state or the first duration of the other state.
} 
modelling from Calvet and Fisher (2001). This earlier theoretical research uses Markov-switching to develop the first time-stationary formulation of multifractal diffusions, and also provides a weakly convergent sequence of discrete filters. We now propose a variant of these filters to directly model financial series in discrete time. In this framework, total volatility is the multiplicative product of a large but finite number of random components. We assume for simplicity that these components are first order Markov and identical except for time scale. The components have identical marginal distributions and differ only in their switching probabilities. The specification is completed by assuming that the progression of switching probabilities is approximately geometric. This parsimonious model delivers long-memory features in volatility, substantial outliers, and a decomposition into components with heterogenous decay rates.

An empirical investigation of four daily exchange rate series shows that our model performs well in comparison with the Student-GARCH(1,1) of Bollerslev (1987), and the Markov-switching GARCH (MS-GARCH) of Klaassen (2002). The choice of alternative processes is guided by several considerations. First, our process easily permits maximum likelihood estimation and analytical multistep forecasting, and we therefore select for comparison models that also have these features. $\operatorname{GARCH}(1,1)$ is an obvious choice because it is a leading model for volatility forecasting. ${ }^{3}$ MS-GARCH provides another useful comparison because it combines regime-shifts with smooth weighting of past shocks, and thus represents a potentially appealing compromise between the two approaches. ${ }^{4}$

The multifractal process compares favorably with $\operatorname{GARCH}(1,1)$ both in- and out-of-sample. Our model has a higher likelihood in-sample for all currencies, and the statistical significance of these differences is confirmed by a HAC-adjusted version of the Vuong (1989) test. Since both models have the same number of parameters, the multifractal is also preferred by standard selection criteria. Analogous results are obtained out-of-sample. While one-day forecasts from the two models perform similarly, the multifractal dominates at longer horizons. The difference is most pronounced for 20 and 50 days. For example, in the case of the British Pound, the 50-day forecasting $R^{2}$ is $27.3 \%$ for our model as compared to $-2.6 \%$ for $\operatorname{GARCH}(1,1)$. Similar gains are obtained for other currencies. The em-

\footnotetext{
${ }^{3}$ See, for example, Akgiray (1989), Andersen and Bollerslev (1998), Hansen and Lunde (2001), Pagan and Schwert (1990), and West and Cho (1995).

${ }^{4}$ The original MS-GARCH process of Gray (1996) does not conveniently permit multistep forecasting, and we therefore consider the variant formulation introduced for this purpose by Klaassen (2002).
} 
pirical evidence thus shows that the multifractal model improves on $\operatorname{GARCH}(1,1)$ both in- and out-of-sample.

The MS-GARCH process gives substantially better fit than $\operatorname{GARCH}(1,1)$ insample. This is partly attributable to a larger number of parameters, and suggests the possibility of overfitting. Using a BIC criterion, the multifractal is statistically indistinguishable from MS-GARCH in-sample for all four currencies. Outof-sample, MS-GARCH is also comparable to the multifractal at short horizons, but substantially dominated at longer horizons. The multifractal is overall the best performing model.

A pure Markov-switching model thus captures the same dynamics that in previous literature have required not only regime-switching but also linear GARCH transitions and a thick-tailed conditional distribution of returns. It is striking that a single mechanism can play all three of these roles so effectively. Our innovation that achieves this surprising economy of modelling technique is based on scale-invariance. We make this principle operational by introducing a pure Markov-switching formulation where scale-invariance helps to specify the parameters and transitions of a high-dimensional state space.

Our paper contributes to the multifractal literature by offering a convenient time-series construction accompanied by effective estimation and testing methods. Calvet, Fisher, and Mandelbrot (1997) introduce the multifractal model of asset returns (MMAR), a class of diffusions that capture the outliers, momentscaling, and long memory in volatility exhibited by many financial time series. While providing an excellent fit to many aspects of financial data, the MMAR uses a combinatorial construction that is not well-suited to econometrics. In particular, regime changes take place at predetermined dates, making the model non-stationary. Early efforts at estimation thus focus on unconditional moments of returns. ${ }^{5}$ Calvet and Fisher (2002) use moment-scaling to develop an estimator and diagnostic tests of the model, while Lux (2001) constructs a GMM estimator based on high-frequency autocovariances. This previous empirical work is constrained in many ways by the non-stationarity of the MMAR. Now using a model that is stationary and a special case of the Hamilton formulation, we develop maximum likelihood estimation, which to the best of our knowledge is new to the

\footnotetext{
${ }^{5}$ The MMAR implies that return moments vary as power functions of the observation interval, which is consistent with many financial series. Calvet, Fisher, and Mandelbrot (1997) and Calvet and Fisher (2002) find evidence of moment-scaling in powers of the absolute value of returns. Further evidence is provided by Andersen, Bollerslev, Diebold and Labys (2001) and BarndorffNielsen and Shephard (2003). See LeBaron (2001) for a discussion of robustness.
} 
literature on multifractal measures and processes.

Section 2 presents the discrete-time model. Section 3 develops the ML estimator and assesses its accuracy in Monte Carlo simulations. Section 4 discusses estimation results for four exchange rates. Section 5 compares our model with alternative processes both in- and out-of-sample. Section 6 concludes. All proofs are in the Appendix.

\section{The Markov-Switching Multifrequency Process}

This section develops a discrete-time Markov process with multi-frequency stochastic volatility. The process has a finite number $\bar{k}$ of latent volatility state variables, each of which corresponds to a different frequency.

\subsection{Stochastic Volatility}

We consider an economic series $X_{t}$ defined in discrete time on the regular grid $t=0,1,2, \ldots, \infty$. In applications, $X_{t}$ will be the log-price of a financial asset or exchange rate. Define the innovations $x_{t} \equiv X_{t}-X_{t-1}$. A common modeling methodology assumes that the system is hit every period by a single shock that progressively phases out over time (e.g., Engle, 1982). We consider instead an economy with $\bar{k}$ components $M_{1, t}, M_{2, t}, \ldots, M_{\bar{k}, t}$, which decay at heterogeneous frequencies $\gamma_{1}, \ldots, \gamma_{\bar{k}}$. Such a model could be very unwieldy as the number $\bar{k}$ becomes very large. We will see, however, that the process can be nonetheless parsimoniously described by a small set of parameters.

We model the innovations $x_{t} \equiv X_{t}-X_{t-1}$ by

$$
x_{t}=\sigma\left(M_{1, t} M_{2, t} \ldots M_{\bar{k}, t}\right)^{1 / 2} \varepsilon_{t},
$$

where the parameter $\sigma$ is a positive constant and the random variables $\varepsilon_{t}$ are IID standard Gaussians $\mathcal{N}(0,1)$. The random multipliers or volatility components $M_{k, t}$ are persistent, non-negative and satisfy $\mathbb{E}\left(M_{k, t}\right)=1$. We consider for simplicity that the multipliers $M_{1, t}, M_{2, t} \ldots M_{\bar{k}, t}$ at a given time $t$ are statistically independent. The parameter $\sigma$ is then equal to the unconditional standard deviation of the innovation $x_{t}$.

Equation (2.1) defines a stochastic volatility model $x_{t}=\sigma_{t} \varepsilon_{t}$ with the multiplicative structure $\sigma_{t}=\sigma\left(M_{1, t} M_{2, t} \ldots M_{\bar{k}, t}\right)^{1 / 2}$. We conveniently stack the period $t$ volatility components multipliers into a vector

$$
M_{t}=\left(M_{1, t}, M_{2, t}, \ldots, M_{\bar{k}, t}\right) .
$$


For any $m=\left(m_{1}, . ., m_{\bar{k}}\right) \in \mathbb{R}^{\bar{k}}$, let $g(m)$ denote the product $\prod_{i=1}^{\bar{k}} m_{i}$. Volatility at time $t$ is then $\sigma_{t}=\sigma\left[g\left(M_{t}\right)\right]^{1 / 2}$.

The properties of volatility are driven by the stochastic dynamics of the vector $M_{t}$. We assume for parsimony that $M_{t}$ is first-order Markov. This facilitates the construction through time of $\left\{x_{t}\right\}$ and permits maximum likelihood estimation. It is then natural to call $M_{t}$ the volatility state vector, and each component $M_{k, t}$ a state variable. The econometrician observes the returns $x_{t}=\sigma\left[g\left(M_{t}\right)\right]^{1 / 2} \varepsilon_{t}$, but not the vector $M_{t}$ itself. The vector $M_{t}$ is therefore latent, and must be inferred recursively by Bayesian updating.

Each $M_{k, t}$ follows a process that is identical except for time scale. Assume that the volatility state vectors have been constructed up to date $t-1$. For each $k \in\{1, . ., \bar{k}\}$, the next period multiplier $M_{k, t}$ is drawn from a fixed distribution $M$ with probability $\gamma_{k}$, and is otherwise equal to its current value: $M_{k, t}=M_{k, t-1}$. The dynamics of $M_{k, t}$ can be summarized as:

$$
\begin{array}{ll}
M_{k, t} \text { drawn from distribution } M & \text { with probability } \gamma_{k} \\
M_{k, t}=M_{k, t-1} & \text { with probability } 1-\gamma_{k} .
\end{array}
$$

The switching events and new draws from $M$ are assumed to be independent across $k$ and $t$. The volatility components $M_{k, t}$ thus differ in their transition probabilities $\gamma_{k}$ but not in their marginal distribution $M$. These features greatly contribute to the parsimony of the model.

The transition probabilities $\gamma \equiv\left(\gamma_{1}, \gamma_{2}, \ldots, \gamma_{\bar{k}}\right)$ are specified as

$$
\gamma_{k}=1-\left(1-\gamma_{1}\right)^{\left(b^{k-1}\right)}
$$

where $\gamma_{1} \in(0,1)$ and $b \in(1, \infty)$. This specification is introduced in Calvet and Fisher (2001) in connection with the discretization of a Poisson arrival process. Since $1-\gamma_{k}=\left(1-\gamma_{1}\right)^{\left(b^{k-1}\right)}$, the logarithms of staying probabilities are exponentially decreasing with $k$. Consider a process with very persistent components, or equivalently a very small parameter $\gamma_{1}$. For small values of $k$, the quantity $\gamma_{1} b^{k-1}$ remains small and the transition probability satisfies:

$$
\gamma_{k} \sim \gamma_{1} b^{k-1}
$$

The transition probabilities of low frequency components grow approximately at geometric rate $b$. At higher frequencies $\left(\gamma_{k} \sim 1\right)$, the rate of increase slows down and condition (2.2) guarantees that the parameter $\gamma_{k}$ remains lower than 1 . In 
empirical applications, it is numerically convenient to estimate parameters of the same magnitude. Since $\gamma_{1}<\ldots<\gamma_{\bar{k}}<1<b$, we choose $\left(\gamma_{\bar{k}}, b\right)$ to specify the set of transition probabilities.

The integer $\bar{k}$ determines the number of volatility frequencies in the model, and we view this choice as a model selection problem. Our approach is consistent with methods commonly employed for $\operatorname{ARMA}(p, q)$ and $\operatorname{GARCH}(p, q)$ models, where estimation is developed for a fixed number of lags and $p$ and $q$ are determined by model selection. The multifractal construction imposes only minimal restrictions on the marginal distribution of the multipliers: $M \geq 0$ and $\mathbb{E}(M)=1$. While this allows flexible parametric or even nonparametric specifications of $M$, this paper focuses on the most parsimonious setup. We assume that $M$ is drawn from a family of unit mean distributions specified by a single parameter $m_{0}$. Useful examples include log-normal distributions: $\ln M \sim \mathcal{N}\left(m_{0},-m_{0}^{2} / 2\right)$, or binomial random variables taking values $m_{0}$ or $2-m_{0}$ with equal probability. The full parameter vector is then

$$
\psi \equiv\left(m_{0}, \sigma, b, \gamma_{\bar{k}}\right) \in \mathbb{R}^{4},
$$

where $m_{0}$ characterizes the distribution of the multipliers, $\sigma$ is the unconditional standard deviation of returns, and $b$ and $\gamma_{\bar{k}}$ define the set of switching probabilities. In Section 3, we will develop and empirically implement the maximum likelihood estimation of this vector.

We find it convenient to call this construct the Markov-Switching Multifractal (or Markov-Switching Multifrequency) process. The notation $\operatorname{MSM}(\bar{k})$ will refer to versions of the model with $\bar{k}$ frequencies. Economic intuition and earlier work suggest that the multiplicative structure (2.1) is appealing to model the high variability and high volatility persistence exhibited by financial time series. When a low level multiplier changes, volatility varies very discontinuously and has strong degree of persistence. In addition, high frequency multipliers introduce substantial outliers.

\subsection{Properties}

The MSM construction permits low frequency regime shifts, and thus long volatility cycles in sample paths. We will see that in exchange rate series, the duration of the most persistent component, $1 / \gamma_{1}$, is typically of the same order as the length of the data. Estimated processes thus tend to generate volatility cycles with periods proportional to the sample size, a property also apparent in the sample paths of long memory processes. 
Long memory is often defined by a hyperbolic decline in the autocovariance function as the lag goes to infinity. Fractionally integrated processes generate such patterns by assuming that an innovation linearly affects future periods at a hyperbolically declining weight. As a result, fractional integration tends to produce smooth volatility processes. By contrast, our approach generates long cycles with a switching mechanism that also gives abrupt volatility changes. The combination of long-memory behavior with sudden volatility movements has a natural appeal for financial econometrics.

In earlier work, we proposed a definition of long memory that applies to continuous time processes defined on a bounded time domain. This definition is based on increments over progressively smaller intervals. Our discrete-time process can also generate a hyperbolic decline in autocovariances for a range of lags. For every integer $n$ and every $q \geq 0$, let $\rho_{q}(n)=\operatorname{Corr}\left(\left|x_{t}\right|^{q},\left|x_{t+n}\right|^{q}\right)$ denote the autocorrelation in levels. We choose a fixed vector $\psi$ and consider the positive parameter $\delta(q)=\log _{b} \frac{\mathbb{E}\left(M^{q}\right)}{\left[\mathbb{E}\left(M^{q / 2}\right)\right]^{2}}$. Consider two arbitrary numbers $\alpha_{1}$ and $\alpha_{2}$ in the open interval $(0,1)$. The set of integers $I_{\bar{k}}=\left\{n: \alpha_{1} \log _{b}\left(b^{\bar{k}}\right) \leq \log _{b} n \leq \alpha_{2} \log _{b}\left(b^{\bar{k}}\right)\right\}$ contains a large range of intermediate lags. We show in the Appendix

Proposition. The autocorrelation in levels satisfies

$$
\sup _{n \in I_{\bar{k}}}\left|\frac{\ln \rho_{q}(n)}{\ln n^{-\delta(q)}}-1\right| \rightarrow 0
$$

as $\bar{k} \rightarrow+\infty$.

MSM thus mimics the hyperbolic autocovariograms $\ln \rho_{q}(n) \sim-\delta(q) \ln n$ exhibited by many financial series (e.g., Dacorogna et al., 1993; Ding and Granger, 1993). This result complements earlier research that has emphasized the difficulty of distinguishing between long memory and structural change in finite samples (e.g., Bhattacharya et al., 1983; Künsch, 1986; Hidalgo and Robinson, 1996; Lobato and Savin, 1997; Diebold and Inoue, 2001). MSM illustrates that a Markovchain regime-switching model can theoretically exhibit one of the defining features of long memory, a hyperbolic decline of the autocovariogram.

A representative return series is illustrated in Figure 1. The graph reveals that multiple switching frequencies help to generate large heterogeneity in volatility levels and substantial outliers. This is notable since the return process has by construction finite moments of every order. We will see that it is nonetheless sufficient to capture the tail properties of the exchange rate data. In Calvet and 
Fisher (2001), we introduced Paretian tails by considering IID shocks of the form $\varepsilon_{t}=Z_{t} \Omega_{t}$, where $\left\{Z_{t}\right\}$ are IID standard Gaussians and $\left\{\Omega_{t}\right\}$ are IID random variables drawn from a Paretian distribution $\Omega$. The random variable $\Omega$ is the limit distribution of a renormalized squared variation of the price process over a fixed horizon, and is fully specified by the existing parameters $m_{0}, b$, and $\gamma_{\bar{k}}$. In the current empirical study, we find it computationally more convenient to consider the Gaussian case because the likelihood is then available in closed-form. The discrete-time process with $\varepsilon_{t}=Z_{t} \Omega_{t}$ is a promising direction for future empirical research.

Another interesting property of our multifractal model is that when $\bar{k} \rightarrow \infty$, the limiting continuous time process lies outside the class of Itô diffusions. The sample paths are continuous but exhibit a high degree of heterogeneity in local behavior, which is characterized by a continuum of local Hölder exponents in any finite time interval. We refer the reader to Calvet and Fisher $(2001,2002)$ for a full development of the continuous-time multifractal limit.

\section{Maximum Likelihood Estimation}

When the multiplier $M$ has a discrete distribution, there exist a finite number of volatility states. Standard filtering methods then provide the likelihood function in closed-form.

\subsection{Updating the State Vector}

We assume in the rest of this paper that the multiplier $M$ takes a finite number of values $b_{m}$. The vector $M_{t}=\left(M_{1, t}, M_{2, t}, \ldots, M_{\bar{k}, t}\right)$ has therefore $d=b_{m}^{\bar{k}}$ possible values $m^{1}, \ldots, m^{d} \in \mathbb{R}_{+}^{\bar{k}}$. The dynamics of the Markov chain $M_{t}$ are characterized by the transition matrix $A=\left(a_{i, j}\right)_{1 \leq i, j \leq d}$ with components $a_{i j}=\mathbb{P}\left(M_{t+1}=m^{j} \mid M_{t}=\right.$ $\left.m^{i}\right){ }^{6}$

Let $n($.$) denote the density of a standard normal. The density of the innovation$ $x_{t}$ conditional on $M_{t}$ is $f_{x_{t}}\left(x \mid M_{t}=m^{i}\right)=\left[\sigma g\left(m^{i}\right)\right]^{-1} n\left[x / \sigma g\left(m^{i}\right)\right]$. Consider the

\footnotetext{
${ }^{6}$ We note that $a_{i j}=\prod_{k=1}^{\bar{k}}\left[\left(1-\gamma_{k}\right) 1_{\left\{m_{k}^{i}=m_{k}^{j}\right\}}+\gamma_{k} \mathbb{P}\left(M=m_{k}^{j}\right)\right]$, where $m_{k}^{i}$ denotes the $m$ th component of vector $m^{i}$, and $1_{\left\{m_{k}^{i}=m_{k}^{j}\right\}}$ is the dummy variable equal to 1 if $m_{k}^{i}=m_{k}^{j}$, and 0 otherwise. In Calvet and Fisher (2001) the transition matrix differs because an innovation to a lower frequency multiplier causes switching in all higher frequency multipliers. Here, we assume that arrival times are independent across frequencies.
} 
conditional probabilities

$$
\Pi_{t}^{j} \equiv \mathbb{P}\left(M_{t}=m^{j} \mid x_{1}, . ., x_{t}\right)
$$

over the unobserved states $m^{1}, \ldots, m^{d}$. We can stack these conditional probabilities in the row vector $\Pi_{t}=\left(\Pi_{t}^{1}, . ., \Pi_{t}^{d}\right) \in \mathbb{R}_{+}^{d}$. Let $\iota=(1, ., 1) \in \mathbb{R}^{d}$. We know that $\Pi_{t} \iota^{\prime}=1$.

Using Bayes' rule, we update the new belief $\Pi_{t+1}$ from the old belief $\Pi_{t}$ and the new innovation $x_{t+1}$. Consider the function

$$
\omega\left(x_{t}\right)=\left[\frac{n\left(x_{t} / \sigma g\left(m^{1}\right)\right)}{\sigma g\left(m^{1}\right)}, \ldots, \frac{n\left(x_{t} / \sigma g\left(m^{d}\right)\right)}{\sigma g\left(m^{d}\right)}\right] .
$$

and let $x * y$ denote the Hadamard product $\left(x_{1} y_{1}, . ., x_{d} y_{d}\right)$ of any $x, y \in \mathbb{R}^{d}$. We show in the Appendix that

$$
\Pi_{t+1}=\frac{\omega\left(x_{t+1}\right) *\left(\Pi_{t} A\right)}{\left[\omega\left(x_{t+1}\right) *\left(\Pi_{t} A\right)\right] \iota^{\prime}} .
$$

This formula expresses the conditional probability $\Pi_{t+1}$ as a function of the observation $x_{t+1}$ and the probability $\Pi_{t}$ calculated in period $t$. These results imply that $\Pi_{t}$ can be computed recursively. In empirical applications, we choose the initial vector $\Pi_{0}$ to be equal to the ergodic distribution of the Markov process. Since the multipliers $\left(M_{1,1}, . ., M_{\bar{k}, 1}\right)$ are independent, the components of $\Pi_{0}$ are uniquely determined by $\Pi_{0}^{j}=\prod_{l=1}^{\bar{k}} \mathbb{P}\left(M=m_{l}^{j}\right)$ for all $j$.

\subsection{The Likelihood Function}

Having solved the conditioning problem, we show in the Appendix that the log likelihood function is

$$
\ln L\left(x_{1}, \ldots, x_{T} ; \psi\right)=\sum_{t=1}^{T} \ln \left[\omega\left(x_{t}\right) \cdot\left(\Pi_{t-1} A\right)\right] .
$$

For a fixed $\bar{k}$, we know that the maximum likelihood estimator (ML) is consistent and asymptotically efficient as $T \rightarrow \infty$. An important difference between MSM and standard Markov switching models stems from the parsimonious parameterization of the transition matrix $A$. This allows us to estimate MSM with reasonable precision even in the presence of a very large state space. While the Expectation Maximization (EM) algorithm proposed in Hamilton (1990) is not directly applicable with constrained transition probabilities, we find that numerical optimization of the likelihood function produces good results. 


\subsection{Small-Sample Properties}

This section assesses the small sample properties of the ML estimator in Monte Carlo simulations. The models investigated have $\bar{k}=8$ frequencies, which is large enough to represent specifications that perform well in later empirical sections. We also restrict attention to the simple binomial specification where the multiplier $M$ takes values $m_{0}$ and $2-m_{0}$ with equal probability.

The four required parameters are thus the binomial value $m_{0}$, the unconditional standard deviation $\sigma$, the frequency growth rate $b$, and the high frequency switching probability $\gamma_{\bar{k}}$. The choice of the unconditional standard deviation is not consequential since it is simply a normalization of size, and we therefore choose $\sigma=1$. The remaining parameters are set to values that are representative of empirical results in later sections. Specifically, all simulations have $b=3$ and $\gamma_{\bar{k}}=0.95$, and the binomial parameter takes one of three values $m_{0} \in\{1.3,1.4,1.5\}$. We also consider three sample lengths $T \in\left\{T_{1}, T_{2}, T_{3}\right\}$, where $T_{1}=2,500, T_{2}=5,000$, and $T_{3}=10,000$.

Table 1 reports the simulation results. Each of the nine columns is based on a different combination of one of the three values of $m_{0}$ with one of the three values of the sample length $T$. For each column, we simulate 400 independent sample paths of the corresponding model and sample length. Maximum likelihood estimation then provides a set of parameter estimates and asymptotic standard errors for each path. ${ }^{7}$ The table has four rows corresponding to each parameter. The first row gives the average point estimate over the simulated paths. The second row is the standard error of these point estimates, or the finite sample standard error (FSSE). The third row gives the root mean squared error (RMSE) of the parameter estimates relative to the true parameter values. ${ }^{8}$ Finally, the average asymptotic standard error (AASE) gives the average over the 400 simulations of the asymptotic standard errors calculated from the information matrix. As sample size becomes large, we expect that the AASE and the FSSE become close.

The results of Table 1 show that maximum likelihood estimation works well. For $m_{0}, \sigma$, and $b$, the biases are small and become negligible as sample size increases. The parameter $m_{0}$ has a low standard error relative to its size and is

\footnotetext{
${ }^{7}$ We start the optimizations at the true parameter values and iterate to convergence once. Preliminary work considered searching for multiple local optima, and although these occasionally exist, they do not significantly affect the means or standard deviations of the reported Monte Carlo results.

${ }^{8}$ The RMSE will always be greater than or equal to the FSSE, and equal to the FSSE only when the average point estimate is identical to the true parameter value.
} 
thus well identified, which is important because this parameter largely determines the variability of volatility. By contrast, the unconditional standard deviation $\sigma$ has standard errors that, although declining as expected with sample length, are roughly ten percent of the true parameter value. We interpret this result as a strength of the model, as it is consistent with the idea that low-frequency variations create considerable uncertainty about long-run averages. The parameter $b$ shows a moderate degree of uncertainty about the amount of spacing between frequencies. Finally, the high-frequency switching probability $\gamma_{\bar{k}}$ is the only parameter that shows more than a small bias. This disappears quickly as sample size increases, and the standard errors are not generally large. Overall the Monte Carlo simulations show that maximum likelihood estimation produces reliable results given the sample sizes considered in subsequent sections. We also note that the convenience and efficiency of maximum likelihood offers significant advantages relative to previous moment-based estimators for multifractal processes.

\section{Empirical Results}

Using a binomial specification for the multiplier $M$, we apply ML estimation to four exchange rate series and obtain preferred specifications with a large number of volatility frequencies.

\subsection{Exchange Rate Data}

The empirical analysis uses daily exchange rate data for the Deutsche Mark (DEM), Japanese Yen (JPY), British Pound (GBP), and Canadian Dollar (CAD), all against the US Dollar. The data consists of daily prices reported at noon by the Federal Reserve Bank of New York. ${ }^{9}$ The fixed exchange rate system broke down in early 1973, and the DEM, JPY and GBP series accordingly begin on 1 June 1973. The CAD series starts a year later (1 June 1974) because the Canadian currency was held essentially at parity with the US Dollar for several months after the demise of Bretton Woods. The Deutsche Mark was replaced by the Euro at the beginning of 1999. The DEM data accordingly ends on 31 December 1998, while the other three series run until 30 June 2002. Overall, the series contains 6,420 observations for the Deutsche Mark, 7,049 observations for the Canadian Dollar, and 7,299 observations for the Yen and the Pound.

\footnotetext{
${ }^{9}$ More specifically, the data consist of buying rates for wire transfers at 12:00 PM Eastern time.
} 
Figure 2 plots the daily returns of each series. Consistent with earlier studies, we observe apparent volatility clustering on a range of frequencies. For each series, Table 2 reports the standard deviation of returns computed over the entire sample and over four subsamples of equal length. We observe that the sample standard deviation can vary quite substantially across subperiods, which is consistent with the low-frequency regime shifts in our model.

\subsection{Estimation Results}

Table 3 reports MLE results for all four currencies. The columns of the table correspond to the number of frequencies $\bar{k}$ varying from 1 to 10 . The first column is thus a standard Markov-switching model with only two possible values for volatility. As $\bar{k}$ increases, the number of states increases at the rate $2^{\bar{k}}$. There are thus over one thousand states when $\bar{k}=10$.

We first examine the DEM data, and note that the multiplier value $\hat{m}_{0}$ tends to decline with $\bar{k}$. This is because with a larger number of volatility components, less variability is required in each individual component to generate the same aggregate amount of stochastic volatility. The estimates of $\hat{\sigma}$ show variability of a different type across $\bar{k}$, with no particular pattern of increasing or decreasing. This is again consistent with the idea that the long-run average of volatility is difficult to identify in a model that permits long volatility cycles. We finally examine the frequency parameters $\hat{\gamma}_{\bar{k}}$ and $\hat{b}$. When $\bar{k}=1$, the parameter $\hat{\gamma}_{\bar{k}}$ indicates a switch in the single multiplier a little less than once every two weeks. As $\bar{k}$ increases, the switching probability of the highest frequency multiplier increases until for large values of $\bar{k}$ a switch occurs almost every day. At the same time, the estimated value $\hat{b}$ decreases steadily with $\bar{k}$. We can use (2.2) to calculate that when $\bar{k}=10$, a switch in the lowest frequency multiplier occurs approximately once every ten years, or about one third the sample size. Thus, as $\bar{k}$ increases, the range of frequencies spreads out while the spacing between frequencies becomes tighter.

The parameter estimates for the other three currencies show many similarities. In all cases, the value of $\hat{m}_{0}$ tends to decrease with $\bar{k}$. We also observe that the values of $\hat{m}_{0}$, and thus the importance of stochastic volatility, are largest for JPY and GBP and smallest for CAD. Consistent with this, variability across $\bar{k}$ in the estimates of $\hat{\sigma}$ is also greatest for JPY and GBP and least for CAD. As $\bar{k}$ increases all currencies also show the highest frequency multiplier switching more often and the spacing between frequencies becoming tighter. The spacing between frequencies is widest for JPY and GBP and tightest for CAD. We correspondingly 
observe that the duration of the lowest frequency multiplier is longest for JPY at approximately three times the sample size, and smallest for CAD at approximately one tenth the sample size. We also note that all asymptotic standard errors are roughly consistent with the magnitudes obtained in our Monte Carlo simulations.

The estimated $\operatorname{MSM}(\bar{k})$ processes generate substantial outliers for large values of $\bar{k}$ despite the fact that they have finite moments of every order. To confirm this, for each data set we use the estimated process with $\bar{k}=10$ frequencies to generate ten thousand paths of the same length as the data. We then compute a Hill tail index $\alpha$ for each simulated path. Basing the index on 100 order statistics, the empirical tail index and the average $\alpha$ in the simulated samples are respectively equal to 4.74 and 4.34 (DEM), 3.91 and 3.75 (JPY), 4.59 and 4.03 (GBP), and 4.40 and 4.79 (CAD). Furthermore, for all currencies we cannot at the $10 \%$ level reject equality of the simulated and empirical tail statistics. This result is caused by the high frequency variations in volatility in the MSM model. With the highest frequency multipliers taking new values almost daily, the distribution of returns is strongly affected by being composed of a mixture of distributions. Even though this mixture of distributions has finite moments of every order, it is more than sufficient to capture the tail characteristics of the data, even in sample sizes as large as almost thirty years of daily observations.

We finally examine the behavior of the log-likelihood function as the number of frequencies $\bar{k}$ increases. For each currency, the likelihood goes up substantially when $\bar{k}$ is low. As $\bar{k}$ gets larger, the likelihood continues to increase in most cases, but at a decreasing rate. The only exception to the monotonic increase in likelihood is the DEM series, for which the likelihood reaches a peak at $\bar{k}=7$. In all other cases the likelihood reaches a maximum at $\bar{k}=10$. This behavior of the likelihood confirms one of the main premises of the multifractal approach. Specifically, volatility fluctuations have a multiplicity of frequencies, and explicitly incorporating a larger number of frequencies results in a better fit. We next examine the statistical significance of these results.

\subsection{Model Selection}

This section investigates whether the estimated $\operatorname{MSM}(\bar{k})$ models have significant differences in likelihoods. We compare two processes $\operatorname{MSM}(\bar{k})$ and $\operatorname{MSM}\left(\bar{k}^{\prime}\right)$, $\bar{k} \neq \bar{k}^{\prime}$, with respective densities $f$ and $g$. The processes are non-nested and have 
log-likelihood difference:

$$
\sqrt{T}\left(\ln L_{T}^{f}-\ln L_{T}^{g}\right)=\frac{1}{\sqrt{T}} \sum_{t=1}^{T} \ln \frac{f\left(x_{t} \mid x_{1}, \ldots, x_{t-1}\right)}{g\left(x_{t} \mid x_{1}, \ldots, x_{t-1}\right)} .
$$

Consider the null hypothesis that the models have identical unconditional expected log-likelihoods. When the observations $\left\{x_{t}\right\}$ are IID, Vuong (1989) shows that the difference $\ln L_{T}^{f}-\ln L_{T}^{g}$ is asymptotically normal under the null. ${ }^{10}$ In addition, the variance of this difference is consistently estimated by the sample variance of the addends $\ln \left[f\left(x_{t} \mid x_{1}, \ldots, x_{t-1}\right) / g\left(x_{t} \mid x_{1}, \ldots, x_{t-1}\right)\right]$. Because it is a strong assumption that the conditional likelihoods are IID, we construct in the Appendix a HAC-adjusted version of the Vuong test. Our discussion is a simplified version of the broader approach recently proposed by Rivers and Vuong (2002).

For each $\bar{k} \in\{1, . ., 9\}$, we test in Table 4 the null hypothesis that $\operatorname{MSM}(\bar{k})$ and MSM(10) fit the data equally well. Since HAC-adjusted tests tend to perform poorly in small samples ${ }^{11}{ }^{11}$ we compute $t$-ratios and one-sided $p$ values using both the original and the HAC-adjusted methods. For $\bar{k} \in\{1,2,3\}$, the log-likelihood difference is significant at the $1 \%$ level in the non-adjusted case (Table $4 A$ ) and at the $5 \%$ level in the HAC case (Table $4 B$ ). This is strong evidence that $\operatorname{MSM}(10)$ significantly outperforms models with 1 to 3 frequencies. For $\bar{k} \in\{4,5\}$, we reject the null at the $5 \%$ (non-adjusted) and $20 \%$ (HAC-adjusted) levels in almost all cases. We view these results as rather substantial evidence that $\operatorname{MSM}(10)$ outperforms models with 4 or 5 frequencies. Lower significance levels are obtained for larger values of $\bar{k}$, and the overall conclusion is that the MSM model works better for larger numbers $\bar{k}$ of frequencies. For this reason and also to maintain consistency in the remaining analysis, we henceforth focus on the $\operatorname{MSM}(\bar{k}=10)$ process for all currencies.

\section{Comparison with Alternative Models}

This Section compares the multifractal model with $\operatorname{GARCH}(1,1)$ and Markovswitching GARCH (MS-GARCH) both in- and out-of-sample.

\footnotetext{
${ }^{10}$ See the Appendix for a more detailed discussion of Vuong tests.

${ }^{11}$ See for example Andrews (1991), Andrews and Monahan (1992), and den Haan and Levin (1997).
} 


\subsection{In-Sample Comparison}

We consider alternative processes of the form $x_{t}=h_{t}^{1 / 2} e_{t}$, where $h_{t}$ is the conditional variance of $x_{t}$ at date $t-1$, and $\left\{e_{t}\right\}$ are IID Student innovations with unit variance and $\nu$ degrees of freedom (d.f.'s). $\operatorname{GARCH}(1,1)$ assumes the recursion $h_{t+1}=\omega+\alpha \varepsilon_{t}^{2}+\beta h_{t}$. MS-GARCH combines short-run autoregressive dynamics with low frequency regime shifts (Gray, 1996; Klaassen, 2002). A latent state $s_{t} \in\{1,2\}$ follows a first-order Markov process with transition probabilities $p_{i j}=\mathbb{P}\left(s_{t+1}=j \mid s_{t}=i\right)$. In every period, the econometrician observes the return $x_{t}$ but not the latent $s_{t}$. For $i=\{1,2\}$, let $h_{t+1}(i)=\operatorname{Var}_{t}\left(x_{t+1} \mid s_{t+1}=i\right)$ denote the variance of $x_{t+1}$ conditional on past returns $\left\{x_{t}\right\}_{s=1}^{t}$ and $s_{t+1}=i$. The quantity $h_{t}$ is latent in every period, and the econometrician can similarly define $\mathbb{E}_{t}\left[h_{t}\left(s_{t}\right) \mid s_{t+1}=i\right]$, the expectation of $h_{t}$ conditional on $s_{t+1}=i$ and past returns. Klaassen (2002) assumes the conditional dynamics:

$$
h_{t+1}(i)=\omega_{i}+\alpha_{i} \varepsilon_{t}^{2}+\beta_{i} \mathbb{E}_{t}\left[h_{t}\left(s_{t}\right) \mid s_{t+1}=i\right] .
$$

The equation conditions volatility on a larger information set than the Gray specification: $h_{t+1}(i)=\omega_{i}+\alpha_{i} \varepsilon_{t}^{2}+\beta_{i} \mathbb{E}_{t-1} h_{t}\left(s_{t}\right)$. We prefer (5.1) for two reasons. First, Klaassen shows that his model has better forecasting performance on three of the exchange rates considered in this paper (DEM, JPY and GBP), and attributes these improvements to finer conditioning. Second, the Klaassen version permits analytical multi-step forecasting, which is a key part of our out-of-sample analysis.

We report in Table 5 the ML estimates of the alternative processes. The coefficient $1 / \nu$ is the inverse of the degrees of freedom in the Student distribution. This is a convenient renormalization that has been frequently used in the literature (e.g., Bollerslev, 1987). Each coefficient $\sigma_{i}, i=1,2$, represents the standard deviation of returns conditional on the volatility state: $\sigma_{i}^{2}=\omega_{i} /\left(1-\alpha_{i}-\beta_{i}\right)$. These coefficients are easier to interpret across models than the intercepts $\omega_{i}$. As shown in Table 6, the multifractal has a higher likelihood than $\operatorname{GARCH}(1,1)$ for all exchange rates in spite of the fact that both processes have the same number of parameters. Note that $\operatorname{GARCH}(1,1)$ approximately matches the likelihoods obtained by MSM with only 3 or 4 frequencies. The multifractal model thus gives an improved fit over $\operatorname{GARCH}(1,1)$ in-sample.

The MS-GARCH model raises different issues because it uses more parameters (9) than either the GARCH or MSM processes (4). Thus, although MS-GARCH has higher likelihoods than either GARCH or MSM, we obtain a different ordering when using the Schwarz BIC criterion. In particular, the multifractal model is 
then indistinguishable from MS-GARCH in the GBP data, and is preferred for DEM and CAD.

As suggested by Vuong (1989), we can also test the statistical significance of differences in the BIC criterion. The last two columns of Table 6 test the GARCH and MS-GARCH models against MSM under this metric. ${ }^{12}$ We again give $p$-values for both the original version of the test as well as a HAC adjusted variant. Under the original version, the in-sample performance of the MSM model over GARCH(1,1) is highly significant for DEM, JPY and GBP, and somewhat significant for CAD. The HAC adjustments produce analogous but slightly weaker results. In comparing the MSM model to MS-GARCH, there is some evidence that the multifractal model is a better performer for DEM and CAD, but the significance is marginal at best. Overall, this analysis suggests that in-sample the multifractal matches the performance MS-GARCH and significantly outperforms $\operatorname{GARCH}(1,1)$.

\subsection{Out-of-Sample Forecasts}

We now investigate the out-of-sample forecasting performance of the competing models. We use forecasting horizons ranging from 1 to 50 days. For each currency, we estimate the three processes on the beginning of the series, and use the last twelve years of data (or approximately half the sample) for our out-of-sample forecasting comparison.

Table 7 reports results of one-day forecasts. The first two columns correspond to the coefficients $\gamma_{0}$ and $\gamma_{1}$ from the Mincer-Zarnowitz OLS regressions $x_{t+1}^{2}=$ $\gamma_{0}+\gamma_{1} \mathbb{E}_{t} x_{t+1}^{2}+u_{t}$ of squared returns on a constant and one-day forecasts. These regressions are common in the financial econometrics literature (e.g., Pagan and Schwert, 1990; West and Cho, 1995; Andersen, Bollerslev, and Meddahi, 2002), and unbiased forecasts would imply $\gamma_{0}=0$ and $\gamma_{1}=1$. We adjust the standard errors of $\gamma_{0}$ and $\gamma_{1}$ for parameter uncertainty as in West and McCracken (1998), and for HAC effects using the weighting and lag selection methodology of Newey and West $(1987,1994)$.

The MSM results show that for each currency, the estimated intercept $\hat{\gamma}_{0}$ is slightly positive and the slope $\hat{\gamma}_{1}$ is slightly lower than 1 . These small biases, however, are not statistically significant. In particular, the hypothesis $\gamma_{0}=0$ is accepted at the $5 \%$ confidence level for all currencies, and $\gamma_{1}=1$ is accepted

\footnotetext{
${ }^{12}$ Note that a BIC test of GARCH against the multifractal model is identical to a likelihood test since both have the same number of parameters.
} 
at the 5\% level for JPY and CAD and at the $1 \%$ level for DEM and GBP. The Mincer-Zarnowitz regressions thus show little evidence of bias in MSM forecasts.

The point estimates $\hat{\gamma}_{0}$ and $\hat{\gamma}_{1}$ are slightly worse with $\operatorname{GARCH}(1,1)$ than with the multifractal. All intercepts are more positive, and the slopes are further away from 1 for three currencies. The biases are also statistically significant. The hypotheses $\gamma_{0}=0$ and $\gamma_{1}=1$ are rejected at the $5 \%$ level in seven out of eight cases. Since $0<\hat{\gamma}_{1}<1$, these results suggest that GARCH forecasts are too variable and can be improved by the linear smoothing $\hat{\gamma}_{0}+\hat{\gamma}_{1} \mathbb{E}_{t} x_{t+1}^{2}$. In contrast, Markovswitching GARCH improves on the out-of-sample performance of $\operatorname{GARCH}(1,1)$. We accept that $\gamma_{0}=0$ at the $5 \%$ confidence level for all currencies, and that $\gamma_{1}=1$ at the $1 \%$ level for DEM, GBP and CAD. Furthermore, the regression estimates are best with MS-GARCH for two currencies (DEM and GBP), and with the multifractal for the other two. The two processes thus seem to perform quite similarly out-of-sample at the one-day horizon. We also report in Table 7 two standard measures of goodness of fit: the Mean Squared Error (MSE) and the restricted $R^{2}$ coefficient. ${ }^{13}$ The multifractal produces the best forecasting $R^{2}$ for DEM and JPY. On the other hand, GARCH produces better results for the GBP and MS-GARCH for the CAD. To summarize the one-day forecast results, the multifractal model appears to slightly dominate $\operatorname{GARCH}(1,1)$ and to give results comparable to MS-GARCH.

Multistep forecasts provide sharper evidence of differences between the three models. We report in Table 8 the results for 20-day forecasts, which are representative of longer horizons. Since our data sets contain only business days, this frequency corresponds to about a month of calendar time. Following Andersen and Bollerslev (1998) and Klaassen (2002), the dependent variable is the sum of squared daily returns $\sum_{s=t}^{t+19} x_{s}^{2}$ over the twenty-day period. Because the average size of returns increases with the sampling interval, the estimated intercepts $\hat{\gamma}_{0}$ are larger in Table 8 than in Table 7 . For each currency, the multifractal produces point estimates of $\gamma_{0}$ and $\gamma_{1}$ that are closest to their preferred values. We also accept the hypotheses $\gamma_{0}=0$ and $\gamma_{1}=1$ in all cases at the $5 \%$ confidence level. By contrast, for the other models each currency gives a strong rejection of either one hypothesis (MS-GARCH) or both (GARCH) at the $5 \%$ confidence level. The reported MSE and $R^{2}$ further confirm that the multifractal provides the best 20-

\footnotetext{
${ }^{13}$ The Mean Squared Error (MSE) quantifies forecast errors in the out-of-sample period: $L^{-1} \sum_{t=T-L+1}^{T}\left(x_{t}^{2}-\mathbb{E}_{t-1} x_{t}^{2}\right)^{2}$. The coefficient of determination is defined by $R^{2}=$ $1-M S E / T S S$, where TSS is the out-of-sample variance of squared returns: TSS = $L^{-1} \sum_{t=T-L+1}^{T}\left(x_{t}^{2}-\sum_{i=T-L+1}^{T} x_{i}^{2} / L\right)^{2}$.
} 
day forecasts for all currencies. The difference is particularly large in the case of the DEM and JPY. The $R^{2}$ coefficient is $13.5 \%$ and $20.5 \%$ respectively for DEM and JPY with the multifractal, while negative values are produced by GARCH and MS-GARCH. ${ }^{14}$

Table 9 reports summary forecasting results and significance tests for horizons of 1, 5, 10, 20 and 50 days. Panel A shows the forecasting $R^{2}$ for each model. For the Mark and the Yen, the multifractal model is quite dominant at the 5-day horizon, and increasingly outperforms other models at longer horizons. For the Pound and the Canadian Dollar, the multifractal is only dominant at horizons of 20 days and higher. Panel B analyzes the statistical significance of these results. At horizons of 50 days, the multifractal model outperforms the other models very significantly for DEM, GBP and JPY, and at a moderate or marginal significance level for CAD. The superior forecasts of the multifractal are also highly significant at horizons of 10 and 20 days for the DEM, and somewhat strong at the 20-day horizons for JPY and GBP.

These results are quite impressive for the multifractal model. Although this is the first forecasting evaluation of MSM and only the simplest binomial specification has been investigated, our process compares well with established models. In particular, $\operatorname{GARCH}(1,1)$ is often viewed as a standard benchmark that is very difficult to outperform in forecasting exercises (e.g., West and Cho, 1995; Andersen and Bollerslev, 1998; Hansen and Lunde, 2001). Nonetheless, our results have shown the multifractal model to match or slightly outperform GARCH and MS-GARCH at short horizons, and in many cases to substantially dominate these models at longer horizons.

\section{Conclusion}

This paper proposes an expanded role for regime-switching in modeling volatility. Traditional approaches, such as Markov-switching ARCH (Cai, 1994; Hamilton and Susmel, 1994) and GARCH (Gray, 1996; Klaassen, 2002), consider separately three categories of volatility dynamics. High-frequency variation is captured by a thick-tailed conditional distribution of returns, mid-range frequencies by smooth $\mathrm{ARCH}$ or GARCH components, and only very low frequencies are modeled with

\footnotetext{
${ }^{14}$ The multifractal yields a higher $R^{2}$ for 20-day returns than for daily returns. This stems from the fact that our measure of 20-day volatility is a sum of daily squared returns $\sum_{s=t}^{t+19} x_{s}^{2}$. As in Andersen and Bollerslev (1998), reduced noise in the volatility measure leads to an increase in explanatory power.
} 
regime-switching. We suggest an alternative approach based on regime-switching at all frequencies. The model is very tightly parameterized in spite of a high dimensional state space. Using four long series of daily exchange rates, we find that MSM matches or dominates the performance of previous models across a range of in-sample and out-of-sample measures. Thus, the primary contribution of the paper is to show that regime-switching can have a much broader scope than previously envisioned. In particular, our pure regime switching model provides a viable alternative to approaches that combine regime-switching, linear volatility dynamics, and flexible tail distributions.

Researchers often focus on applications of immediate practical value when assessing statistical models. We have similarly shown that MSM does well by several standard measures of performance. From a theoretical perspective, good econometric descriptions are also useful as the first step in explanation. Whereas the standard approach requires the understanding of three statistical phenomena, MSM offers the possibility of a much more parsimonious elucidation. More specifically, this paper invites the theorist to determine the economic mechanism causing the self-similar form of regime-switching exhibited by financial series.

Returning to the more immediate contributions of our work, this is the first paper to develop and use a comprehensive econometric toolkit to estimate and test multifractal processes. We develop the first maximum likelihood estimator for multifractal processes, which is based on the filter developed by Hamilton (1989). This estimator works well in Monte Carlo simulations, and the application to exchange rates leads to several observations. First, the likelihood function of MSM increases monotonically as we add volatility components. This finding is important because it confirms substantial heterogeneity in volatility persistence, which is one of the primary motivations of the multifractal approach. Consistent with intuition, the spacing of volatility components across frequencies becomes tighter and the contribution of individual volatility components becomes smaller as the number of components increases.

We then compared the multifractal model with Student GARCH and MSGARCH, which are chosen because of demonstrated good performance with exchange rate data under a variety of metrics. Like MSM, these models conveniently permit maximum likelihood estimation and analytical forecasting. In-sample, the likelihood is significantly higher for the multifractal than for GARCH even though these processes have the same number of parameters. We use a BIC criterion to compare goodness-of-fit of MS-GARCH and MSM, and find no significant difference between the two models. Out-of-sample evidence further validates the multi- 
fractal process. MSM matches or slightly outperforms GARCH and MS-GARCH at short horizons, and in many cases substantially dominates these models at longer horizons. The multifractal model thus appears to be a serious contender among volatility models, and well-deserving of further empirical and theoretical investigation. 


\section{Appendix}

\subsection{Autocorrelogram}

Consider a sequence of processes with fixed parameter vector $\psi=\left(m_{0}, \sigma, b, \gamma^{*}\right)$. Note in particular that $\gamma_{\bar{k}}=\gamma^{*}$ for all $\bar{k}$. For any integer $n \geq 0$ and real $q \in$ $[0, \infty)$, it is convenient to define $K_{q}(n)=\mathbb{E}\left(\left|x_{t}\right|^{q}\left|x_{t+n}\right|^{q}\right) /\left[\mathbb{E}\left(\left|x_{t}\right|^{2 q}\right)\right]$ and $c_{q}=$ $\left[\mathbb{E}\left(\left|\varepsilon_{t}\right|^{q}\right)\right]^{2} /\left[\mathbb{E}\left(\left|\varepsilon_{t}\right|^{2 q}\right)\right]$. Multipliers in different stages of the cascade are statistically independent. The definition of returns, $x_{t}=\sigma\left(M_{1, t} M_{2, t} \ldots M_{\bar{k}, t}\right)^{1 / 2} \varepsilon_{t}$ and $x_{t+n}=$ $\sigma\left(M_{1, t+n} M_{2, t+n} \ldots M_{\bar{k}, t+n}\right)^{1 / 2} \varepsilon_{t+n}$, implies

$$
K_{q}(n)=c_{q}\left[\mathbb{E}\left(M^{q}\right)\right]^{-\bar{k}} \prod_{k=1}^{\bar{k}} \mathbb{E}\left(M_{k, t}^{q / 2} M_{k, t+n}^{q / 2}\right) .
$$

Note that $\mathbb{E}\left(M_{k, t}^{q / 2} M_{k, t+n}^{q / 2}\right)=\mathbb{E}\left(M^{q}\right)\left(1-\gamma_{k}\right)^{n}+\left[\mathbb{E}\left(M^{q / 2}\right)\right]^{2}\left[1-\left(1-\gamma_{k}\right)^{n}\right]$ or equivalently

$$
\mathbb{E}\left(M_{k, t}^{q / 2} M_{k, t+n}^{q / 2}\right)=\left[\mathbb{E}\left(M^{q / 2}\right)\right]^{2} \quad\left[1+a_{q}\left(1-\gamma_{k}\right)^{n}\right],
$$

where $a_{q}=\mathbb{E}\left(M^{q}\right) /\left[\mathbb{E}\left(M^{q / 2}\right)\right]^{2}-1$. Since $1-\gamma_{k}=\left(1-\gamma_{\bar{k}}\right)^{b^{k-\bar{k}}}$ and $\gamma_{\bar{k}}=\gamma^{*}$, we obtain

$$
\ln \frac{K_{q}(n)}{c_{q}}=\sum_{k=1}^{\bar{k}} \ln \frac{1+a_{q}\left(1-\gamma^{*}\right)^{n b^{k-\bar{k}}}}{1+a_{q}} .
$$

As $k$ increases from 1 to $\bar{k}$, the expression $\left(1-\gamma^{*}\right)^{n b^{k-\bar{k}}}$ declines from $\left(1-\gamma^{*}\right)^{n b^{1-\bar{k}}}$ to $\left(1-\gamma^{*}\right)^{n}$. The maximum $\left(1-\gamma^{*}\right)^{n b^{1-\bar{k}}}$ is close to 1 and the minimum $\left(1-\gamma^{*}\right)^{n}$ is close to 0 when $b^{\bar{k}} / n$ and $n$ are large. Intermediate values are observed when $\left(1-\gamma^{*}\right)^{n b^{k-\bar{k}}} \approx 1-\gamma^{*}$, or equivalently $k \approx \log _{b}\left(b^{\bar{k}} / n\right)$. Let $i(n)$ denote the unique integer such that $i(n) \leq \log _{b}\left(b^{\bar{k}} / n\right)<i(n)+1$. We anticipate that

$$
\ln \frac{K_{q}(n)}{c_{q}} \approx \sum_{k=i(n)+1}^{\bar{k}} \ln \frac{1}{1+a_{q}}=-[\bar{k}-i(n)] \ln \left(1+a_{q}\right),
$$

and thus $\ln K_{q}(n) \approx-\left(\log _{b} n\right) \ln \left(1+a_{q}\right)=-\delta(q) \ln n$.

To formalize this intuition, consider the interval $I_{\bar{k}}=\left\{n: \alpha_{1} \log _{b}\left(b^{\bar{k}}\right) \leq\right.$ $\left.\log _{b} n \leq \alpha_{2} \log _{b}\left(b^{\bar{k}}\right)\right\}$. Note that $\log _{b}\left(b^{\bar{k}} / n\right) \geq\left(1-\alpha_{2}\right) \log _{b}\left(b^{\bar{k}}\right)$ for all $n \in I_{\bar{k}}$. We henceforth assume that $\bar{k}$ is sufficiently large so that $i(n) \geq b \forall n \in I_{\bar{k}}$. Consider an arbitrary sequence of strictly positive integers $j(n)$ monotonically diverging to 
$+\infty$. The precise definition of $j(n)$ is temporarily postponed. Let

$$
u_{n}=j(n) \ln \left(1+a_{q}\right)+\sum_{k=i(n)-j(n)+1}^{i(n)+j(n)} \ln \frac{1+a_{q}\left(1-\gamma^{*}\right)^{n b^{k-\bar{k}}}}{1+a_{q}} .
$$

By (7.1), $\ln \left[K_{q}(n) / c_{q}\right]$ can be decomposed into four components:

$$
\begin{aligned}
\ln \frac{K_{q}(n)}{c_{q}}= & -[\bar{k}-i(n)] \ln \left(1+a_{q}\right)+\sum_{k=1}^{i(n)-j(n)} \ln \frac{1+a_{q}\left(1-\gamma^{*}\right)^{n b^{k-\bar{k}}}}{1+a_{q}} \\
& +u_{n}+\sum_{k=i(n)+j(n)+1}^{\bar{k}} \ln \left[1+a_{q}\left(1-\gamma^{*}\right)^{n b^{k-\bar{k}}}\right] .
\end{aligned}
$$

We successively examine each component on the righ-hand side.

- The first component is between $-\delta(q)(\ln n+\ln b)$ and $-\delta(q) \ln n$.

- The second component contains terms $\left(1-\gamma^{*}\right)^{n b^{k-\bar{k}}}$ that are bounded below by $\left(1-\gamma^{*}\right)^{n b^{i(n)-j(n)-\bar{k}}}$. The definition of $i(n)$ implies $n b^{i(n)-\bar{k}} \leq 1$ and thus

$$
\left|\sum_{k=1}^{i(n)-j(n)} \ln \frac{1+a_{q}\left(1-\gamma^{*}\right)^{n b^{k-\bar{k}}}}{1+a_{q}}\right| \leq i(n) \ln \frac{1+a_{q}}{1+a_{q}\left(1-\gamma^{*}\right)^{b^{-j(n)}}} .
$$

By standard concavity arguments, we infer $\ln \frac{1+a_{q}}{1+a_{q}\left(1-\gamma^{*}\right)^{b^{-j(n)}}} \leq a_{q}[1-(1-$ $\left.\left.\gamma^{*}\right)^{b^{-j(n)}}\right]$ and $1-e^{b^{-j(n)} \ln \left(1-\gamma^{*}\right)} \leq b^{-j(n)}\left|\ln \left(1-\gamma^{*}\right)\right|$. The second component of (7.2) is therefore bounded by $i(n) b^{-j(n)} a_{q}\left|\ln \left(1-\gamma^{*}\right)\right|$.

- The third component, $u_{n}$, contains terms $1+a_{q}\left(1-\gamma^{*}\right)^{n b^{k-\bar{k}}}$ that are between 1 and $1+a_{q}$. Hence $\left|u_{n}\right| \leq j(n) \ln \left(1+a_{q}\right) \leq a_{q} j(n)$.

- The fourth component is positive and bounded above by

$$
a_{q} \sum_{k=i(n)+j(n)+1}^{\bar{k}}\left(1-\gamma^{*}\right)^{n b^{k-\bar{k}}} \leq a_{q} \sum_{k=0}^{\infty}\left(1-\gamma^{*}\right)^{b^{k} n b^{i(n)+j(n)+1-\bar{k}}} .
$$

We check that $n b^{i(n)+j(n)+1-\bar{k}} \geq 1$ and $b^{k} \geq k(b-1)$. The fourth component is therefore bounded above by $a_{q} \sum_{k=0}^{\infty}\left(1-\gamma^{*}\right)^{k(b-1)}=\frac{a_{q}}{1-\left(1-\gamma^{*}\right)^{b-1}}$. 
This establishes that

$$
\left|\frac{\ln K_{q}(n)}{\ln n^{-\delta(q)}}-1\right| \leq \frac{c_{q}^{*}+a_{q} j(n)+a_{q} i(n) b^{-j(n)}\left|\ln \left(1-\gamma^{*}\right)\right|}{\delta(q) \ln n},
$$

where $c_{q}^{*}=\delta(q) \ln b+\left|\ln c_{q}\right|+a_{q} /\left[1-\left(1-\gamma^{*}\right)^{(b-1)}\right]$. We now choose a sequence $j(n)$ such that the right-hand side of the inequality converges to 0. More specifically, consider the unique integer such that ${ }^{15} j(n) \leq 2 \log _{b} i(n)<j(n)+1$. It is easy to check that $i(n) b^{-j(n)}=b^{\log _{b} i(n)-j(n)} \leq 1$ and $j(n) \leq 2 \log _{b}\left(\log _{b} b^{\bar{k}}\right)=2 \log _{b} \bar{k}$. For all $n \in I_{\bar{k}}$, the quantity $\left|\frac{\ln K_{q}(n)}{\ln n^{-\delta(q)}}-1\right|$ is therefore bounded above by

$$
\eta_{\bar{k}}=\frac{1}{\bar{k} \delta(q) \alpha_{1} \ln b}\left[2 a_{q} \log _{b} \bar{k}+c_{q}^{*}+a_{q}\left|\ln \left(1-\gamma^{*}\right)\right|\right]
$$

which is independent of $n$. We infer that $\sup _{n \in I_{\bar{k}}}\left|\frac{\ln K_{q}(n)}{\ln n^{-\delta(q)}}-1\right| \rightarrow 0$ as $\bar{k} \rightarrow+\infty$.

Finally, it is easy to show that the autocorrelation $\rho_{q}(n)$ satisfies

$$
1 \leq \frac{K_{q}(n)}{\rho_{q}(n)}=\frac{1-c_{q}\left(1+a_{q}\right)^{-\bar{k}}}{1-c_{q}\left(1+a_{q}\right)^{-\bar{k}} / K_{q}(n)} \leq \frac{1}{1-c_{q}\left(1+a_{q}\right)^{-\bar{k}} / K_{q}(n)} .
$$

Equation (7.3) implies that for all $n \in I_{\bar{k}}, \log _{b} K_{q}(n) \geq-\delta(q)\left(1+\eta_{\bar{k}}\right) \alpha_{2} \bar{k}$, and thus

$$
\log _{b}\left[K_{q}(n) /\left(1+a_{q}\right)^{-\bar{k}}\right] \geq \bar{k} \delta(q)\left(1-\alpha_{2}-\alpha_{2} \eta_{\bar{k}} / \bar{k}\right) .
$$

Combining (7.4) and (7.5), we conclude that $\sup _{n \in I_{\bar{k}}}\left|\ln \frac{K_{q}(n)}{\rho_{q}(n)}\right| \rightarrow 0$ and thus that the Proposition holds.

\subsection{Bayesian Updating}

Denote the set of past observations $\mathcal{I}_{t} \equiv\left\{x_{s}\right\}_{s=1}^{t}$. We note that the conditional probability $\Pi_{t+1}^{j}=\mathbb{P}\left(M_{t+1}=m^{j} \mid \mathcal{I}_{t}, x_{t+1}\right)$ satisfies

$$
\Pi_{t+1}^{j}=f_{x_{t+1}}\left(x_{t+1} \mid M_{t+1}=m^{j}\right) \mathbb{P}\left(M_{t+1}=m^{j} \mid \mathcal{I}_{t}\right) / f_{x_{t+1}}\left(x_{t+1} \mid \mathcal{I}_{t}\right),
$$

which can be rewritten as

$$
\Pi_{t+1}^{j}=\frac{n\left[x_{t+1} / \sigma g\left(m^{j}\right)\right]\left(\sum_{i=1}^{d} a_{i j} \Pi_{t}^{i}\right)}{\sigma g\left(m^{j}\right) f_{x_{t}}\left(x_{t+1} \mid \mathcal{I}_{t}\right)} .
$$

This implies (3.1) since $\sum_{j} \Pi_{t+1}^{j}=1$.

\footnotetext{
${ }^{15}$ We check that when $\bar{k}$ is large enough, $1 \leq j(n) \leq i(n)$ and $j(n)+i(n) \leq \bar{k}$ for all $n \in I_{\bar{k}}$.
} 


\subsection{Likelihood Function}

We know that $L\left(x_{1}, \ldots, x_{T} ; \psi\right)=\sum_{t=1}^{T} \ln f\left(x_{t} \mid x_{1}, \ldots, x_{t-1}\right)$. Bayes' rule implies that

$$
\begin{aligned}
f\left(x_{t} \mid x_{1}, \ldots, x_{t-1}\right) & =\sum_{i=1}^{d} \mathbb{P}\left(M_{t}=m^{i} \mid x_{1}, \ldots, x_{t-1}\right) f\left(x_{t} \mid M_{t}=m^{i}\right) \\
& =\sum_{i=1}^{d} \mathbb{P}\left(M_{t}=m^{i} \mid x_{1}, \ldots, x_{t-1}\right) \frac{1}{\sigma g\left(m^{i}\right)} n\left(\frac{x}{\sigma g\left(m^{i}\right)}\right)
\end{aligned}
$$

and thus $f\left(x_{t} \mid x_{1}, \ldots, x_{t-1}\right)=\omega\left(x_{t}\right) \cdot\left(\Pi_{t-1} A\right)$.

\subsection{HAC-Adjusted Vuong Test}

We consider the probability space $\left(\Omega, \mathcal{F}, \mathbb{P}^{0}\right)$ and a stochastic process $\left\{x_{t}\right\}_{t=-\infty}^{+\infty}$. Each $x_{t}$ is a random variable taking values on the real line. For every $t$, it is convenient to consider the vector of past values $X_{t-1}=\left\{x_{s}\right\}_{s=-\infty}^{t-1}$. The econometrician directly observes a finite number of realizations of $x_{t}$, but ignores the true data generating process. She instead considers two competing families of models specified by their conditional densities $\mathcal{M}_{f}=\left\{f\left(x_{t} \mid X_{t-1}, \theta\right) ; \theta \in \Theta\right\}$ and $\mathcal{M}_{g}=\left\{g\left(x_{t} \mid X_{t-1}, \gamma\right) ; \gamma \in \Gamma\right\}$. These families may or may not contain the true data generating process. The pseudo true value $\theta^{*}$ specifies the model in $\mathcal{M}_{f}$ with the optimal Kullback-Leibler Information Criterion:

$$
\theta^{*}=\arg \max _{\theta \in \Theta} \quad \mathbb{E}^{0}\left[\ln f\left(x_{t} \mid X_{t-1}, \theta\right)\right] .
$$

The pseudo true value $\gamma^{*}$ is similarly defined.

Consider the log-likelihood functions:

$$
L_{T}^{f}(\theta) \equiv \sum_{t=1}^{T} \ln f\left(x_{t} \mid X_{t-1}, \theta\right), \quad L_{T}^{g}(\gamma) \equiv \sum_{t=1}^{T} \ln g\left(x_{t} \mid X_{t-1}, \gamma\right) .
$$

By definition, the MLE estimators $\hat{\theta}_{T}$ and $\hat{\gamma}_{T}$ maximize the functions $L_{T}^{f}(\theta)$ and $L_{T}^{g}(\gamma)$. The corresponding FOCs are

$$
\frac{\partial L_{T}^{f}}{\partial \theta}\left(\hat{\theta}_{T}\right)=0, \quad \frac{\partial L_{T}^{g}}{\partial \theta}\left(\hat{\gamma}_{T}\right)=0 .
$$


We now examine the likelihood ratio

$$
L R_{T}\left(\hat{\theta}_{T}, \hat{\gamma}_{T}\right)=L_{T}^{f}\left(\hat{\theta}_{T}\right)-L_{T}^{g}\left(\hat{\gamma}_{T}\right)=\sum_{t=1}^{T} \ln \frac{f\left(x_{t} \mid X_{t-1}, \hat{\theta}_{T}\right)}{g\left(x_{t} \mid X_{t-1}, \hat{\gamma}_{T}\right)} .
$$

By equation (7.7), a second order expansion of $L R_{T}$ implies that $\frac{1}{\sqrt{T}} L R_{T}\left(\hat{\theta}_{T}, \hat{\gamma}_{T}\right)=$ $\frac{1}{\sqrt{T}} L R_{T}\left(\theta^{*}, \gamma^{*}\right)+o_{p}(1)$, and thus

$$
\frac{1}{\sqrt{T}} L R_{T}\left(\hat{\theta}_{T}, \hat{\gamma}_{T}\right)=\frac{1}{\sqrt{T}} \sum_{t=1}^{T} \ln \frac{f\left(x_{t} \mid X_{t-1}, \theta^{*}\right)}{g\left(x_{t} \mid X_{t-1}, \gamma^{*}\right)}+o_{p}(1) .
$$

Let $a_{t}=\ln \left[f\left(x_{t} \mid X_{t-1}, \theta^{*}\right) / g\left(x_{t} \mid X_{t-1}, \gamma^{*}\right)\right]$ and $\hat{a}_{t}=\ln \left[f\left(x_{t} \mid X_{t-1}, \hat{\theta}_{T}\right) / g\left(x_{t} \mid X_{t-1}, \hat{\gamma}_{T}\right)\right]$.

When the observations $x_{t}$ are IID, the addends $a_{t}$ are also IID. If the models $f$ and $g$ have equal Kullback-Leibler Information criterion, the CLT implies

$$
\frac{1}{\sqrt{T}} L R_{T}\left(\hat{\theta}_{T}, \hat{\gamma}_{T}\right) \stackrel{d}{\rightarrow} \mathcal{N}\left(0, \sigma_{*}^{2}\right)
$$

where $\sigma_{*}^{2}=\operatorname{Var}\left(a_{t}\right)$. The variance is consistently estimated by the sample variance of $\left\{\hat{a}_{t}\right\}$.

In the non-IID case, we need to adjust for the correlation in the addends $a_{t}$. Let

$$
\sigma_{T}^{2}=\frac{1}{T} \sum_{s=1}^{T} \sum_{t=1}^{T} \mathbb{E}\left(a_{s} a_{t}\right)
$$

We know that $\frac{1}{\sqrt{T}} L R_{T}\left(\hat{\theta}_{T}, \hat{\gamma}_{T}\right)=\sigma_{T} Z+o_{p}(1)$, where $Z$ is a standard Gaussian. Following Newey-West (1987), we estimate $\sigma_{T}$ by

$$
\hat{\sigma}_{T}^{2}=\hat{\Omega}_{0}+2 \sum_{j=1}^{m_{T}} w(j, m) \hat{\Omega}_{j},
$$

where $\hat{\Omega}_{j}=\sum_{t=j+1}^{T} \hat{a}_{t} \hat{a}_{t-j} / T$ denotes the sample covariance of $\left\{\hat{a}_{t}\right\}$, and $w(j, m)=$ $1-j /(m+1)$ is the Bartlett weight. We choose $m_{T}$ using the automatic lag selection method of Newey and West (1994). 


\section{References}

[1] Akgiray, V. (1989), Conditional Heteroskedasticity in Time Series of Stock Returns: Evidence and Forecasts, Journal of Business 62, 55-80.

[2] Albert, J., and Chibb, S. (1993), Bayes Inference via Gibbs Sampling of Autoregressive Time Series Subject to Markov Means and Variance Shifts, Journal of Business and Economic Statistics 11, 1-15.

[3] Andersen, T.G., and Bollerslev, T. (1998), Answering the Skeptics: Yes, Standard Volatility Models Do Provide Accurate Forecasts, International Economic Review 39, 885-905.

[4] Andersen, T. G., Bollerslev, T., Diebold, F. X., and Labys, P. (2001), The Distribution of Realized Exchange Rate Volatility, Journal of the American Statistical Association 96,42-55.

[5] Andersen, T. G., Bollerslev, T., and Meddahi, N. (2002), Analytic Evaluation of Volatility Forecasts, CIRANO Working Paper 2002s-90.

[6] Andrews, D. W. K. (1991), Heteroskedasticity and Autocorrelation Consistent Covariance Matrix Estimation, Econometrica 59, 817-854.

[7] Andrews, D. W. K., and Monahan, J. C. (1992), An Improved Heteroskedasticity and Autocorrelation Consistent Covariance Matrix Estimator, Econometrica 60, 953-966.

[8] Barndorff-Nielsen, O., and Shephard, N. (2003), Realized Power Variation and Stochastic Volatility, Bernoulli, forthcoming.

[9] Baum, L. E., Ted, P., George, S., and Weiss, N. (1980), A Maximization Technique Occurring in the Statistical Analysis of Probabilistic Functions of Markov Chains, Annals of Mathematical Statistics 41, 164-171.

[10] Bhattacharya, R. N., Gupta, V. K., and Waymire, E. (1983), The Hurst Effect under Trends, Journal of Applied Probability 20, 649-662.

[11] Bollen, N., Gray, S. and Whaley, R. (2000), Regime Switching in Foreign Exchange Rates: Evidence from Currency Option Prices, Journal of Econometrics 94, 239-276. 
[12] Bollerslev, T. (1987), A Conditional Heteroskedastic Time Series Model for Speculative Prices and Rates of Return, Review of Economics and Statistics 69, 542-547.

[13] Cai, J. (1994), A Markov Model of Unconditional Variance in ARCH, Journal of Business and Economic Statistics 12, 309-316.

[14] Calvet, L., and Fisher, A. (2001), Forecasting Multifractal Volatility, Journal of Econometrics 105, 27-58.

[15] Calvet, L., and Fisher, A. (2002), Multifractality in Asset Returns: Theory and Evidence, Review of Economics and Statistics 84, 381-406.

[16] Calvet, L., Fisher, A., and Mandelbrot, B. B. (1997), Cowles Foundation Discussion Papers No. 1164-1166, Yale University. Papers available from http://www.ssrn.com.

[17] Dacorogna, M., Müller, U., Nagler, R., Olsen, R., and Pictet, O. (1993), A Geographical Model for the Daily and Weekly Seasonal Volatility in the Foreign Exchange Market, Journal of International Money and Finance 12, 413-438.

[18] den Haan, W. J., and Levin, A. (1997), "A Practitioner's Guide to Robust Covariance Matrix Estimation", in G. Maddala and C. Rao Eds., Handbook of Statistics: Robust Inference, Vol. 15, New York: Elsevier.

[19] Diebold. F. X., and Inoue, A. (2001), Long Memory and Regime Switching, Journal of Econometrics 105, 27-58.

[20] Diebold, F. X., Lee, J. H., and Weinbach, G. C. (1994), Regime Switching with Time-Varying Transition Probabilities, in C. Hargreaves ed. Nonstationary Time Series Analysis and Cointegration, Oxford University Press.

[21] Ding, Z., Granger, C., and Engle, R. (1993), A Long Memory Property of Stock Market Returns and a New Model, Journal of Empirical Finance 1, 83-106.

[22] Durland, J., and McCurdy, T. (1994), Duration-Dependent Transitions in a Markov Model of US GNP Growth, Journal of Business and Economic Statistics 12, 279-288. 
[23] Engle, R. F. (1982), Autoregressive Conditional Heteroscedasticity with Estimates of the Variance of United Kingdom Inflation, Econometrica 50, 9871007.

[24] Filardo, A. J. (1994), Business Cycle Phases and their Transitional Dynamics, Journal of Business and Economic Statistics 12, 299-308.

[25] Garcia, R. (1998), Asymptotic Null Distribution of the Likelihood Ratio Test in Markov Switching Models, International Economic Review 39, 763-788.

[26] Garcia, R., and Perron, P. (1996), An Analysis of the Real Interest Rate under Regime Shifts, Review of Economics and Statistics 78, 111-125.

[27] Ghysels, E., Harvey, A. C., and Renault, E. (1996), Stochastic Volatility, in: G. S. Maddala and C. R. Rao Eds., Handbook of Statistics, Vol. 14, 119-191. Amsterdam: North-Holland.

[28] Gray, S. (1996), Modeling the Conditional Distribution of Interest Rates as a Regime-Switching Process, Journal of Financial Economics 42, $27-62$.

[29] Hamilton, J. D. (1988), Rational Expectations Econometric Analysis of Changes in Regimes: An Investigation of the Term Structure of Interest Rates, Journal of Economic Dynamics and Control 12, 385-423.

[30] Hamilton, J. D. (1989), A New Approach to the Economic Analysis of Nonstationary Time Series and the Business Cycle, Econometrica 57, 357-84.

[31] Hamilton, J. D. (1990), Analysis of Time Series Subject to Change in Regime, Journal of Econometrics 45, 39-70.

[32] Hamilton, J. D., and Lin, G. (1996), Stock Market Volatility and the Business Cycle, Journal of Applied Econometrics 11, 573-593.

[33] Hamilton, J. D., and Perez-Quiros, G. (1996), What Do the Leading Indicators Lead?, Journal of Business 69, 27-49.

[34] Hamilton, J. D. and B. Raj (2002), New Directions in Business Cycle Research and Financial Analysis, Empirical Economics 27, 149-162.

[35] Hamilton, J. D. and R. Susmel (1994), Autoregressive Conditional Heteroskedasticity and Changes in Regime, Journal of Econometrics 64, 307333. 
[36] Hansen, B. (1992), The Likelihood Ratio Test under Non-Standard Conditions: Testing the Markov-Switching Model of GNP, Journal of Applied Econometrics 7, 561-582.

[37] Hansen, P., and Lunde, A. (2001), "A Forecast Comparison of Volatility Models: Does Anything Beat a GARCH(1,1)?", Working Paper, Brown University.

[38] Hidalgo, J., and Robinson, P. M. (1996), Testing for Structural Change in a Long-Memory Environment, Journal of Econometrics 70, 159-174.

[39] Kim, C. J. (1994), Dynamic Linear Models with Markov-Switching, Journal of Econometrics 60, 1-22.

[40] Kim, C. J., and Nelson, C. R. (1999), State-Space Models with RegimeSwitching, Cambridge, Mass.: MIT Press.

[41] Klaassen, F. (2002), Improving GARCH Volatility Forecasts with RegimeSwitching GARCH, Empirical Economics 27, 363-394.

[42] Künsch, H. R. (1986), Discrimination between Monotonic Trends and LongRange Dependence, Journal of Applied Probability 23, 1025-1030.

[43] LeBaron, B. (2001), Stochastic Volatility as a Simple Generator of Apparent Financial Power Laws and Long Memory, Quantitative Finance 1, 621-631.

[44] Lindgren, G. (1978), Markov Regime Models for Mixed Distributions and Switching Regressions, Scandinavian Journal of Statistics 5, 81-91.

[45] Lobato, I. N., and Savin, N. E. (1997), Real and Spurious Long-Memory Properties of Stock-Market Data, Journal of Business and Economic Statistics 16, 261-283.

[46] Lux, T. (2001), "The Multifractal Model of Asset Returns: Simple Moment and GMM Estimation", Working Paper, Kiel University.

[47] Maheu, J., and McCurdy, T. (2000), Volatility Dynamics under DurationDependent Mixing, Journal of Empirical Finance 7, 345-372

[48] Newey, W., and West, K. (1987), A Simple, Positive Semi-Definite, Heteroskedasticity and Autocorrelation Consistent Covariance Matrix, Econometrica 55, 703-708. 
[49] Newey, W., and West, K. (1994), Automatic Lag Selection in Covariance Matrix Estimation, Review of Economic Studies 61, 631-654.

[50] Pagan, A., and Schwert, W. (1990), Alternative Models for Conditional Stock Volatility, Journal of Econometrics 45, 267-290.

[51] Perez-Quiros, G., and Timmerman, A. (2000), Firm Size and Cyclical Variations in Stock Returns, Journal of Finance 55, 1229-1262.

[52] Rivers, D., and Vuong, Q. (2002), Model Selection Tests for Nonlinear Dynamic Models, Econometrics Journal 5 (1), 1-39.

[53] Shephard, N. (1994), Partial Non-Gaussian State Space, Biometrika 81, 115131.

[54] Vuong, Q. (1989), Likelihood Ratio Tests for Model Selection and Non-Nested Hypotheses, Econometrica 57, 307-333.

[55] West, K., and Cho, D. (1995), The Predictive Ability of Several Models of Exchange Rate Volatility, Journal of Econometrics 69, 367-391.

[56] West, K., and McCracken, M. (1998), Regression-Based Tests of Predictive Ability, International Economic Review 39, 817-840. 
TABle 1. - Monte Carlo Mle Results

\begin{tabular}{|c|c|c|c|c|c|c|c|c|c|}
\hline & \multicolumn{3}{|c|}{$\bar{m}_{0}=1.3$} & \multicolumn{3}{|c|}{$m_{0}=1.4$} & \multicolumn{3}{|c|}{$\bar{c}_{0}=1.5$} \\
\hline & $T_{1}$ & $T_{2}$ & $T_{3}$ & $T_{1}$ & $T_{2}$ & $T_{3}$ & $T_{1}$ & $T_{2}$ & $T_{3}$ \\
\hline $\bar{m}_{s i m}$ & 1.288 & 1.293 & 1.297 & 1.392 & 1.393 & 1.397 & 1.494 & 1.494 & 1.497 \\
\hline FSSE & $(0.026)$ & $(0.018)$ & $(0.012)$ & $(0.031)$ & $(0.019)$ & $(0.015)$ & $(0.032)$ & $(0.025)$ & $(0.015)$ \\
\hline RMSE & $(0.029)$ & $(0.019)$ & $(0.012)$ & $(0.032)$ & $(0.021)$ & $(0.015)$ & $(0.033)$ & $(0.025)$ & $(0.016)$ \\
\hline AASE & $(0.019)$ & $(0.013)$ & $(0.010)$ & $(0.018)$ & $(0.014)$ & $(0.011)$ & $(0.019)$ & $(0.014)$ & $(0.011)$ \\
\hline $\bar{\sigma}_{\text {sim }}$ & 1.014 & 1.004 & 0.999 & 1.031 & 1.011 & 1.005 & 1.026 & 1.017 & 1.006 \\
\hline FSSE & $(0.167)$ & $(0.102)$ & $(0.073)$ & $(0.221)$ & $(0.147)$ & $(0.091)$ & $(0.255)$ & $(0.224)$ & $(0.105)$ \\
\hline RMSE & $(0.167)$ & $(0.102)$ & $(0.073)$ & $(0.223)$ & $(0.148)$ & $(0.092)$ & $(0.256)$ & $(0.225)$ & $(0.105)$ \\
\hline AASE & $(0.072)$ & $(0.056)$ & $(0.046)$ & $(0.091)$ & $(0.075)$ & $(0.057)$ & $(0.111)$ & $(0.088)$ & $(0.067)$ \\
\hline $\bar{\gamma}_{s i m}$ & 0.867 & 0.908 & 0.934 & 0.907 & 0.935 & 0.940 & 0.924 & 0.938 & 0.944 \\
\hline FSSE & $(0.164)$ & $(0.113)$ & $(0.068)$ & $(0.111)$ & $(0.069)$ & $(0.047)$ & $(0.087)$ & $(0.055)$ & $(0.037)$ \\
\hline RMSE & $(0.184)$ & $(0.120)$ & $(0.070)$ & $(0.119)$ & $(0.070)$ & $(0.048)$ & $(0.091)$ & $(0.056)$ & $(0.037)$ \\
\hline AASE & $(0.181)$ & $(0.114)$ & $(0.071)$ & $(0.110)$ & $(0.068)$ & $(0.048)$ & $(0.078)$ & $(0.052)$ & $(0.036)$ \\
\hline $\bar{b}_{\text {sim }}$ & 2.853 & 2.942 & 2.988 & 3.052 & 2.938 & 2.973 & 3.054 & 2.987 & 2.979 \\
\hline FSSE & $(0.934)$ & $(0.670)$ & $(0.416)$ & $(0.963)$ & $(0.480)$ & $(0.363)$ & $(0.735)$ & $(0.565)$ & $(0.316)$ \\
\hline RMSE & $(0.945)$ & $(0.673)$ & $(0.416)$ & $(0.964)$ & $(0.484)$ & $(0.364)$ & $(0.737)$ & $(0.565)$ & $(0.317)$ \\
\hline AASE & $(0.677)$ & $(0.471)$ & $(0.331)$ & $(0.599)$ & $(0.367)$ & $(0.264)$ & $(0.476)$ & $(0.323)$ & $(0.225)$ \\
\hline
\end{tabular}

Notes: This table is based on $J=400$ simulated paths for each column. All simulations are based on a multifractal process with $\bar{k}=8$. The columns are distinguished by combinations of $m_{0} \in\{1.3,1.4,1.5\}$ and sample lengths of $T_{1}=2500, T_{2}=5000$, and $T_{3}=10000$. Parameters that are fixed across all simulations are $\sigma=1, \gamma_{k}=0.95$, and $b=3$. These parameters provide a reasonable approximation to values that are estimated on exchange rate data in future sections of the paper. In each set of four rows, the first row is the average MLE parameter value over the $J$ simulated paths. In the remaining rows, FSSE denotes finite sample standard error, RMSE the root mean squared error, and AASE the average asymptotic standard error. To derive AASE, the asymptotic variance is calculated for each $j \in\{1, . ., J\}$ path from the inverse of the information matrix and the average is taken over the $J$ simulations. 
TABLE 2. - FX RETURN VARIABILITY

\begin{tabular}{lcccccc}
\hline \hline & \multicolumn{5}{c}{ Standard Deviation of Returns } \\
\cline { 2 - 6 } & Entire & \multicolumn{5}{c}{ By Subperiod } \\
Sample & & 1 & 2 & 3 & 4 \\
\cline { 2 - 6 } \cline { 4 - 7 } JPY & 0.664 & & 0.587 & 0.716 & 0.708 & 0.635 \\
GBP & 0.657 & & 0.545 & 0.640 & 0.646 & 0.775 \\
CAD & 0.607 & & 0.486 & 0.724 & 0.699 & 0.473 \\
& 0.274 & & 0.220 & 0.255 & 0.284 & 0.327 \\
\hline
\end{tabular}

Notes: For each data set, the first column shows the standard deviation of returns over the entire sample period. In the next four columns, each data series is broken into quarters and the same statistic is calculated for each subperiod. The results show that the variability of return variance is substantial even at very low frequencies. 
TABLE 3. - MAXIMUM Likelihood Results

\begin{tabular}{|c|c|c|c|c|c|c|c|c|c|c|}
\hline & $k=1$ & 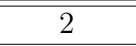 & 3 & 4 & 5 & $\overline{66}$ & $\overline{7}$ & 8 & 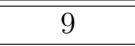 & 10 \\
\hline \multicolumn{11}{|c|}{ Deutsche Mark / US Dollar } \\
\hline \multirow[t]{2}{*}{$\hat{m}_{0}$} & 1.654 & 1.590 & 1.555 & 1.492 & 1.462 & 1.413 & 1.380 & 1.353 & 1.351 & 1.326 \\
\hline & $(0.013)$ & $(0.012)$ & $(0.013)$ & $(0.013)$ & $(0.012)$ & $(0.013)$ & $(0.012)$ & $(0.011)$ & $(0.013)$ & $(0.015)$ \\
\hline \multirow[t]{2}{*}{$\hat{\sigma}$} & 0.682 & 0.651 & 0.600 & 0.572 & 0.512 & 0.538 & 0.547 & 0.550 & 0.674 & 0.643 \\
\hline & $(0.012)$ & $(0.018)$ & $(0.014)$ & $(0.016)$ & $(0.018)$ & $(0.026)$ & $(0.021)$ & $(0.025)$ & $(0.035)$ & $(0.073)$ \\
\hline \multirow[t]{2}{*}{$\hat{\gamma}_{\bar{k}}$} & 0.075 & 0.107 & 0.672 & 0.714 & 0.751 & 0.858 & 0.932 & 0.974 & 0.966 & 0.959 \\
\hline & $(0.011)$ & $(0.022)$ & $(0.151)$ & $(0.096)$ & $(0.106)$ & $(0.128)$ & $(0.071)$ & $(0.042)$ & $(0.065)$ & $(0.066)$ \\
\hline \multirow[t]{2}{*}{$\hat{b}$} & - & 8.01 & 21.91 & 10.42 & 7.89 & 5.16 & 4.12 & 3.38 & 3.29 & 2.70 \\
\hline & & $(2.58)$ & $(7.30)$ & $(1.92)$ & ( 1.31$)$ & $(0.76)$ & $(0.48)$ & $(0.36)$ & $(0.47)$ & $(0.36)$ \\
\hline \multirow[t]{2}{*}{$\ln L$} & -5920.86 & -5782.96 & -5731.78 & -5715.31 & -5708.25 & -5706.91 & -5704.48 & -5704.77 & -5704.86 & -5705.09 \\
\hline & & & & Japan & se Yen / I & S Dollar & & & & \\
\hline \multirow[t]{2}{*}{$\hat{m}_{0}$} & 1.797 & 1.782 & 1.693 & 1.654 & 1.640 & 1.573 & 1.565 & 1.513 & 1.475 & 1.448 \\
\hline & $(0.011)$ & $(0.009)$ & $(0.010)$ & $(0.010)$ & $(0.010)$ & $(0.010)$ & $(0.010)$ & $(0.010)$ & $(0.010)$ & $(0.011)$ \\
\hline \multirow[t]{2}{*}{$\hat{\sigma}$} & 0.630 & 0.538 & 0.566 & 0.462 & 0.709 & 0.642 & 0.518 & 0.514 & 0.486 & 0.461 \\
\hline & $(0.011)$ & $(0.009)$ & $(0.017)$ & $(0.013)$ & $(0.023)$ & $(0.023)$ & $(0.018)$ & $(0.020)$ & $(0.026)$ & $(0.036)$ \\
\hline \multirow[t]{2}{*}{$\hat{\gamma}_{\bar{k}}$} & 0.199 & 0.345 & 0.312 & 0.697 & 0.778 & 0.899 & 0.897 & 0.975 & 0.995 & 0.998 \\
\hline & $(0.019)$ & $(0.033)$ & $(0.054)$ & $(0.080)$ & $(0.076)$ & $(0.060)$ & $(0.057)$ & $(0.034)$ & $(0.010)$ & $(0.006)$ \\
\hline \multirow[t]{2}{*}{$\hat{b}$} & - & 134.20 & 12.46 & 15.58 & 16.03 & 8.07 & 7.46 & 5.65 & 4.43 & 3.76 \\
\hline & & $(48.27)$ & ( 2.18$)$ & ( 2.67$)$ & ( 2.67) & ( 1.03$)$ & $(0.89)$ & $(0.78)$ & $(0.53)$ & $(0.45)$ \\
\hline \multirow[t]{2}{*}{$\ln L$} & -6451.80 & -6102.18 & -5959.72 & -5900.67 & -5882.93 & -5871.35 & -5867.88 & -5863.20 & -5863.01 & -5862.68 \\
\hline & & & & Briti & Pound & $S$ Dollar & & & & \\
\hline \multirow[t]{2}{*}{$\hat{m}_{0}$} & 1.716 & 1.671 & 1.648 & 1.609 & 1.579 & 1.534 & 1.503 & 1.461 & 1.428 & 1.403 \\
\hline & $(0.012)$ & $(0.011)$ & $(0.011)$ & $(0.011)$ & $(0.011)$ & $(0.012)$ & $(0.012)$ & $(0.011)$ & $(0.011)$ & $(0.009)$ \\
\hline \multirow[t]{2}{*}{$\hat{\sigma}$} & 0.609 & 0.590 & 0.513 & 0.467 & 0.421 & 0.468 & 0.389 & 0.384 & 0.374 & 0.370 \\
\hline & $(0.009)$ & $(0.011)$ & $(0.016)$ & $(0.016)$ & $(0.017)$ & $(0.019)$ & $(0.014)$ & $(0.015)$ & $(0.022)$ & $(0.022)$ \\
\hline \multirow[t]{2}{*}{$\hat{\gamma}_{\bar{k}}$} & 0.110 & 0.222 & 0.278 & 0.645 & 0.637 & 0.784 & 0.811 & 0.958 & 0.964 & 0.982 \\
\hline & $(0.017)$ & $(0.034)$ & $(0.052)$ & $(0.080)$ & $(0.075)$ & $(0.078)$ & $(0.083)$ & $(0.052)$ & $(0.043)$ & $(0.031)$ \\
\hline \multirow[t]{2}{*}{$\hat{b}$} & - & 19.90 & 14.29 & 12.51 & 11.02 & 8.32 & 6.72 & 5.23 & 4.08 & 3.45 \\
\hline & & $(5.19)$ & ( 2.58$)$ & $(2.00)$ & $(1.74)$ & $(1.15)$ & & $(0.69)$ & $(0.41)$ & $(0.32)$ \\
\hline \multirow[t]{2}{*}{$\ln L$} & -5960.18 & -5724.37 & -5622.73 & -5570.02 & -5537.80 & -5523.64 & -5516.89 & -5515.37 & -5515.28 & -5514.94 \\
\hline & & & & Canadi & Dollar & IS Dollar & & & & \\
\hline \multirow[t]{2}{*}{$\hat{m}_{0}$} & 1.646 & 1.556 & 1.474 & 1.435 & 1.386 & 1.374 & 1.338 & 1.319 & 1.296 & 1.278 \\
\hline & $(0.012)$ & $(0.012)$ & $(0.014)$ & $(0.015)$ & $(0.012)$ & $(0.013)$ & $(0.012)$ & $(0.016)$ & $(0.013)$ & $(0.012)$ \\
\hline \multirow[t]{2}{*}{$\hat{\sigma}$} & 0.280 & 0.278 & 0.293 & 0.263 & 0.251 & 0.295 & 0.282 & 0.262 & 0.259 & 0.262 \\
\hline & $(0.005)$ & $(0.006)$ & $(0.014)$ & $(0.009)$ & $(0.010)$ & $(0.011)$ & $(0.013)$ & $(0.017)$ & $(0.015)$ & $(0.021)$ \\
\hline \multirow[t]{2}{*}{$\hat{\gamma}_{\bar{k}}$} & 0.064 & 0.109 & 0.129 & 0.171 & 0.441 & 0.524 & 0.593 & 0.594 & 0.631 & 0.644 \\
\hline & $(0.009)$ & $(0.016)$ & $(0.040)$ & $(0.062)$ & $(0.153)$ & $(0.128)$ & $(0.145)$ & $(0.151)$ & $(0.155)$ & $(0.158)$ \\
\hline \multirow[t]{2}{*}{$\hat{b}$} & - & 10.92 & 4.76 & 3.95 & 4.02 & 4.08 & 3.11 & 2.72 & 2.35 & 2.11 \\
\hline & & ( 3.12$)$ & ( 1.15$)$ & $(0.83)$ & $(0.76)$ & $(0.58)$ & ( 0.39$)$ & & $(0.25)$ & $(0.18)$ \\
\hline $\ln L$ & -271.01 & -129.80 & -105.16 & -91.32 & -88.41 & -84.73 & -84.03 & -83.40 & -83.06 & -83.00 \\
\hline
\end{tabular}

Notes: This table shows maximum likelihood estimation results for the binomial multifractal model for all four exchange rate series. Columns correspond to the number of frequencies $\bar{k}$ in the estimated model. The likelihood function increases monotonically in the number of volatility frequencies for all data sets except DEM, which obtains a maximum at $\bar{k}=7$. Asymptotic standard errors are in parenthesis. 
TABlE 4. - Multifractal Model Selection

\begin{tabular}{lccccccccc}
\hline \hline & $k=1$ & 2 & 3 & 4 & 5 & 6 & 7 & 8 & 9 \\
\hline Mark & -8.655 & -5.523 & -2.972 & -1.858 & -0.688 & -0.733 & 0.341 & 0.204 & 0.337 \\
& $(0.000)$ & $(0.000)$ & $(0.001)$ & $(0.032)$ & $(0.246)$ & $(0.232)$ & $(0.633)$ & $(0.581)$ & $(0.632)$ \\
Yen & -13.067 & -8.406 & -5.342 & -3.154 & -2.156 & -1.192 & -1.108 & -0.180 & -0.162 \\
& $(0.000)$ & $(0.000)$ & $(0.000)$ & $(0.001)$ & $(0.016)$ & $(0.117)$ & $(0.134)$ & $(0.429)$ & $(0.436)$ \\
Pound & -11.810 & -8.337 & -6.267 & -4.360 & -2.984 & -1.334 & -0.408 & -0.149 & -0.236 \\
& $(0.000)$ & $(0.000)$ & $(0.000)$ & $(0.000)$ & $(0.001)$ & $(0.089)$ & $(0.342)$ & $(0.441)$ & $0.407)$ \\
Canada & -8.475 & -4.421 & -3.289 & -1.795 & -2.108 & -0.862 & -0.825 & -0.472 & -0.158 \\
& $(0.000)$ & $(0.000)$ & $(0.000)$ & $(0.036)$ & $(0.017)$ & $(0.194)$ & $(0.205)$ & $(0.318)$ & $(0.437)$ \\
\hline \multirow{2}{*}{ Mark } & & & B. HAC Adjusted Vuong & Test & & & \\
& -4.285 & -3.033 & -1.683 & -1.101 & -0.402 & -0.424 & 0.197 & 0.120 & 0.194 \\
Yen & $(0.000)$ & $(0.001)$ & $(0.046)$ & $(0.135)$ & $(0.344)$ & $(0.336)$ & $(0.578)$ & $(0.548)$ & $(0.577)$ \\
& -5.219 & -4.262 & -2.865 & -1.645 & -1.224 & -0.648 & -0.663 & -0.105 & -0.098 \\
Pound & $(0.000)$ & $(0.000)$ & $(0.002)$ & $(0.050)$ & $(0.111)$ & $(0.259)$ & $(0.254)$ & $(0.458)$ & $(0.461)$ \\
& -3.788 & -2.804 & -2.803 & -2.195 & -1.759 & -0.779 & -0.242 & -0.088 & -0.137 \\
Canada & $(0.000)$ & $(0.003)$ & $(0.003)$ & $(0.014)$ & $(0.039)$ & $(0.218)$ & $(0.404)$ & $(0.465)$ & $(0.446)$ \\
& -4.237 & -2.383 & -1.789 & -1.019 & -1.150 & -0.480 & -0.445 & -0.276 & -0.091 \\
& $(0.000)$ & $(0.009)$ & $(0.037)$ & $(0.154)$ & $(0.125)$ & $(0.316)$ & $(0.328)$ & $(0.391)$ & $(0.464)$ \\
\hline
\end{tabular}

Notes: This table reports $t$-ratios and one-sided $p$-values for the log-likelihood difference of the model in each column against the multifractal with ten frequencies. Panel A uses the Vuong (1989) methodology and Panel B adjusts for heteroskedasticity and autocorrelation using Newey and West (1987, 1994). A low $p$-value indicates that the corresponding model would be rejected in favor of the multifractal with ten frequencies. 


\section{TABle 5. - Alternative Processes}

\begin{tabular}{|c|c|c|c|c|c|c|c|c|c|c|}
\hline & \multirow[b]{2}{*}{$1 / \nu$} & \multicolumn{4}{|c|}{ Regime 1} & \multicolumn{4}{|c|}{ Regime 2} & \multirow[b]{2}{*}{$\ln L$} \\
\hline & & $\sigma_{1}$ & $\alpha_{1}$ & $\beta_{1}$ & $p_{11}$ & $\sigma_{2}$ & $\alpha_{2}$ & $\beta_{2}$ & $p_{22}$ & \\
\hline \multicolumn{11}{|c|}{ Deutsche Mark / US Dollar } \\
\hline GARCH & $\begin{array}{l}0.1929 \\
(0.011)\end{array}$ & $\begin{array}{l}1.5539 \\
(0.405)\end{array}$ & $\begin{array}{l}0.0879 \\
(0.009)\end{array}$ & $\begin{array}{l}0.9108 \\
(0.009)\end{array}$ & & & & & & -5730.52 \\
\hline MS-GARCH & $\begin{array}{l}0.2041 \\
(0.011)\end{array}$ & $\begin{array}{l}1.0749 \\
(0.288)\end{array}$ & $\begin{array}{l}0.2048 \\
(0.023)\end{array}$ & $\begin{array}{l}0.7896 \\
(0.024)\end{array}$ & $\begin{array}{c}0.9998 \\
(0.0003)\end{array}$ & $\begin{array}{l}1.3145 \\
(0.282)\end{array}$ & $\begin{array}{l}0.0718 \\
(0.010)\end{array}$ & $\begin{array}{l}0.9241 \\
(0.011)\end{array}$ & $\begin{array}{c}0.9999 \\
(0.0002)\end{array}$ & -5694.78 \\
\hline \multicolumn{11}{|c|}{ Japanese Yen / US Dollar } \\
\hline GARCH & $\begin{array}{c}0.2290 \\
(0.0002)\end{array}$ & $\begin{array}{l}0.1638 \\
(0.059)\end{array}$ & $\begin{array}{l}0.0652 \\
(0.006)\end{array}$ & $\begin{array}{l}0.9348 \\
(0.006)\end{array}$ & & & & & & -5965.07 \\
\hline MS-GARCH & $\begin{array}{l}0.2632 \\
(0.012)\end{array}$ & $\begin{array}{l}0.4443 \\
(0.137)\end{array}$ & $\begin{array}{l}0.3420 \\
(0.040)\end{array}$ & $\begin{array}{l}0.6500 \\
(0.040)\end{array}$ & $\begin{array}{c}0.9999 \\
(0.0002)\end{array}$ & $\begin{array}{l}0.9639 \\
(0.121)\end{array}$ & $\begin{array}{c}0.0650 \\
(0.010)\end{array}$ & $\begin{array}{l}0.9227 \\
(0.013)\end{array}$ & $\begin{array}{c}0.9999 \\
(0.0002)\end{array}$ & -5833.59 \\
\hline \multicolumn{11}{|c|}{ British Pound / US Dollar } \\
\hline GARCH & $\begin{array}{c}0.2007 \\
(0.0077)\end{array}$ & $\begin{array}{c}0.2365 \\
(0.070)\end{array}$ & $\begin{array}{c}0.0681 \\
(0.0046)\end{array}$ & $\begin{array}{c}0.9319 \\
(0.0046)\end{array}$ & & & & & & -5562.00 \\
\hline MS-GARCH & $\begin{array}{l}0.2202 \\
(0.009)\end{array}$ & $\begin{array}{l}0.8423 \\
(0.013)\end{array}$ & $\begin{array}{l}0.3653 \\
(0.053)\end{array}$ & $\begin{array}{l}0.6051 \\
(0.056)\end{array}$ & $\begin{array}{c}0.9860 \\
(0.005)\end{array}$ & $\begin{array}{l}0.9343 \\
(0.012)\end{array}$ & $\begin{array}{c}0.0587 \\
(0.008)\end{array}$ & $\begin{array}{l}0.9365 \\
(0.008)\end{array}$ & $\begin{array}{c}0.9986 \\
(0.0003)\end{array}$ & -5492.44 \\
\hline \multicolumn{11}{|c|}{ Canadian Dollar / US Dollar } \\
\hline GARCH & $\begin{array}{l}0.1528 \\
(0.037)\end{array}$ & $\begin{array}{l}0.3108 \\
(0.008)\end{array}$ & $\begin{array}{l}0.0810 \\
(0.008)\end{array}$ & $\begin{array}{l}0.9108 \\
(0.010)\end{array}$ & & & & & & -96.03 \\
\hline MS-GARCH & $\begin{array}{l}0.1385 \\
(0.011)\end{array}$ & $\begin{array}{l}0.2046 \\
(0.035)\end{array}$ & $\begin{array}{l}0.0584 \\
(0.009)\end{array}$ & $\begin{array}{l}0.9361 \\
(0.010)\end{array}$ & $\begin{array}{c}0.9896 \\
(0.004)\end{array}$ & $\begin{array}{l}0.2972 \\
(0.025)\end{array}$ & $\begin{array}{l}0.2587 \\
(0.074)\end{array}$ & $\begin{array}{l}0.2925 \\
(0.215)\end{array}$ & $\begin{array}{l}0.9415 \\
(0.023)\end{array}$ & -73.51 \\
\hline
\end{tabular}

Notes: This table shows maximum likelihood estimation results for alternative processes for the four exchange rate series. Asymptotic standard errors are in parenthesis. For the $\operatorname{GARCH}(1,1)$ model, the parameter estimates for JPY/USD and GBP/USD are on the boundary of the restriction $\alpha+\beta \leq 1-\epsilon$, where $\epsilon=10^{-5}$. 


\section{TABLE 6. - In-SAmple Model Comparison}

\begin{tabular}{|c|c|c|c|c|c|}
\hline & $\begin{array}{c}\text { No. of } \\
\text { Parameters }\end{array}$ & $\ln L$ & $\mathrm{BIC}$ & $\begin{array}{c}\text { BIC } \\
\text { vs. } \mathrm{Mu}\end{array}$ & $\begin{array}{l}\text { value } \\
\text { ifractal }\end{array}$ \\
\hline & & & & $\begin{array}{l}\text { Vuong } \\
(1989)\end{array}$ & $\begin{array}{l}\text { HAC } \\
\text { Adj }\end{array}$ \\
\hline \multicolumn{6}{|c|}{ Deutsche Mark / US Dollar } \\
\hline Binomial Multifractal & 4 & -5705.09 & 1.7830 & & \\
\hline GARCH & 4 & -5730.52 & 1.7910 & 0.005 & 0.071 \\
\hline MS-GARCH & 9 & -5694.78 & 1.7866 & 0.140 & 0.248 \\
\hline \multicolumn{6}{|c|}{ Japanese Yen / US Dollar } \\
\hline Binomial Multifractal & 4 & -5862.68 & 1.6115 & & \\
\hline GARCH & 4 & -5965.07 & 1.6396 & 0.000 & 0.008 \\
\hline MS-GARCH & 9 & -5833.59 & 1.6097 & 0.619 & 0.572 \\
\hline \multicolumn{6}{|c|}{ British Pound / US Dollar } \\
\hline Binomial Multifractal & 4 & -5514.94 & 1.5162 & & \\
\hline GARCH & 4 & -5562.00 & 1.5291 & 0.004 & 0.070 \\
\hline MS-GARCH & 9 & -5492.44 & 1.5162 & 0.505 & 0.503 \\
\hline \multicolumn{6}{|c|}{ Canadian Dollar / US Dollar } \\
\hline Binomial Multifractal & 4 & -83.00 & 0.0286 & & \\
\hline GARCH & 4 & -96.03 & 0.0323 & 0.072 & 0.200 \\
\hline MS-GARCH & 9 & -73.51 & 0.0322 & 0.092 & 0.235 \\
\hline
\end{tabular}

Notes: This table summarizes information about in-sample goodness of fit for the three models. The Bayesian Information Criterion is given by $B I C=T^{-1}(-2 \ln L+N P \ln T)$. The sample lengths are 6419 for DEM/USD, 7298 for JPY/USD and GBP/USD, and 7048 for CAD/USD. The last two columns give $p$-values from a test that the corresponding model dominates the multifractal model by the BIC criterion. The first value uses the Vuong (1989) methodology, and the second value adjusts the test for heteroskedasticity and autocorrelation. A low $p$-value indicates that the corresponding model would be rejected in favor of the multifractal model. 


\section{TABLE 7. - ONE-DAY FORECASTS}

\begin{tabular}{|c|c|c|c|c|}
\hline & \multicolumn{2}{|c|}{$\begin{array}{c}\text { Mincer- } \\
\text { Zarnowitz }\end{array}$} & \multicolumn{2}{|c|}{$\begin{array}{c}\text { Restricted } \\
\gamma_{0}=0, \gamma_{1}=1\end{array}$} \\
\hline & $\gamma_{0}$ & $\gamma_{1}$ & MSE & $R^{2}$ \\
\hline \multicolumn{5}{|c|}{ Deutsche Mark / US Dollar } \\
\hline Binomial Multifractal & $\begin{array}{c}0.098 \\
(0.072)\end{array}$ & $\begin{array}{c}0.703 \\
(0.126)\end{array}$ & 0.7263 & 0.041 \\
\hline GARCH & $\begin{array}{c}0.153 \\
(0.061)\end{array}$ & $\begin{array}{c}0.622 \\
(0.105)\end{array}$ & 0.7304 & 0.035 \\
\hline MS-GARCH & $\begin{array}{c}0.042 \\
(0.080)\end{array}$ & $\begin{array}{c}0.740 \\
(0.130)\end{array}$ & 0.7296 & 0.037 \\
\hline \multicolumn{5}{|c|}{ Japanese Yen / US Dollar } \\
\hline Binomial Multifractal & $\begin{array}{c}0.028 \\
(0.090)\end{array}$ & $\begin{array}{c}0.772 \\
(0.117)\end{array}$ & 1.6053 & 0.053 \\
\hline GARCH & $\begin{array}{c}0.172 \\
(0.075)\end{array}$ & $\begin{array}{c}0.668 \\
(0.105)\end{array}$ & 1.6137 & 0.048 \\
\hline MS-GARCH & $\begin{array}{c}0.080 \\
(0.084)\end{array}$ & $\begin{array}{c}0.709 \\
(0.109)\end{array}$ & 1.6141 & 0.048 \\
\hline \multicolumn{5}{|c|}{ British Pound / US Dollar } \\
\hline Binomial Multifractal & $\begin{array}{c}0.053 \\
(0.049)\end{array}$ & $\begin{array}{l}0.715 \\
(0.100)\end{array}$ & 0.5081 & 0.057 \\
\hline GARCH & $\begin{array}{c}0.085 \\
(0.044)\end{array}$ & $\begin{array}{c}0.751 \\
(0.098)\end{array}$ & 0.4980 & 0.076 \\
\hline MS-GARCH & $\begin{array}{c}0.017 \\
(0.051)\end{array}$ & $\begin{array}{c}0.814 \\
(0.108)\end{array}$ & 0.4997 & 0.072 \\
\hline \multicolumn{5}{|c|}{ Canadian Dollar / US Dollar } \\
\hline Binomial Multifractal & $\begin{array}{c}0.015 \\
(0.016)\end{array}$ & $\begin{array}{c}0.905 \\
(0.156)\end{array}$ & 0.0345 & 0.051 \\
\hline GARCH & $\begin{array}{c}0.033 \\
(0.012)\end{array}$ & $\begin{array}{l}0.679 \\
(0.111)\end{array}$ & 0.0348 & 0.042 \\
\hline MS-GARCH & $\begin{array}{c}0.025 \\
(0.013)\end{array}$ & $\begin{array}{c}0.785 \\
(0.124)\end{array}$ & 0.0344 & 0.055 \\
\hline
\end{tabular}

Notes: This table gives out of sample forecasting results for the three models. The first two columns correspond to parameter estimates from the Mincer-Zarnowitz OLS regression $e_{t+1}^{2}=\gamma_{0}+\gamma_{1} E_{t}\left(e_{t+1}^{2}\right)+u_{t}$. For an unbiased forecast we expect $\gamma_{0}=0$ and $\gamma_{1}=1$. Asymptotic standard errors in parenthesis are corrected for heteroskedasticity and autocorrelation using the method of Newey and West $(1987,1994)$ and for parameter uncertainty using the method of West and McCracken (1998). MSE is the mean square forecast error, and $R^{2}$ is one less the MSE divided by the sum of squared demeaned squared returns in the out of sample period. 


\section{TABLE 8. - TWENTY-DAY FORECASTS}

\begin{tabular}{|c|c|c|c|c|}
\hline & \multicolumn{2}{|c|}{$\begin{array}{c}\text { Mincer- } \\
\text { Zarnowitz }\end{array}$} & \multicolumn{2}{|c|}{$\begin{array}{c}\text { Restricted } \\
\gamma_{0}=0, \gamma_{1}=1\end{array}$} \\
\hline & $\gamma_{0}$ & $\gamma_{1}$ & MSE & $R^{2}$ \\
\hline \multicolumn{5}{|c|}{ Deutsche Mark / US Dollar } \\
\hline Binomial Multifractal & $\begin{array}{c}1.749 \\
(1.649)\end{array}$ & $\begin{array}{c}0.706 \\
(0.150)\end{array}$ & 37.12 & 0.135 \\
\hline GARCH & $\begin{array}{c}4.474 \\
(1.108)\end{array}$ & $\begin{array}{c}0.443 \\
(0.092)\end{array}$ & 49.24 & -0.147 \\
\hline MS-GARCH & $\begin{array}{c}1.934 \\
(1.577)\end{array}$ & $\begin{array}{c}0.568 \\
(0.118)\end{array}$ & 50.66 & -0.180 \\
\hline \multicolumn{5}{|c|}{ Japanese Yen / US Dollar } \\
\hline Binomial Multifractal & $\begin{array}{l}-1.248 \\
(2.160)\end{array}$ & $\begin{array}{c}0.909 \\
(0.155)\end{array}$ & 76.95 & 0.205 \\
\hline GARCH & $\begin{array}{c}5.311 \\
(1.233)\end{array}$ & $\begin{array}{c}0.488 \\
(0.086)\end{array}$ & 99.15 & -0.024 \\
\hline MS-GARCH & $\begin{array}{c}2.148 \\
(1.776)\end{array}$ & $\begin{array}{c}0.573 \\
(0.108)\end{array}$ & 103.29 & -0.067 \\
\hline \multicolumn{5}{|c|}{ British Pound / US Dollar } \\
\hline Binomial Multifractal & $\begin{array}{c}0.330 \\
(1.114)\end{array}$ & $\begin{array}{c}0.792 \\
(0.120)\end{array}$ & 27.35 & 0.250 \\
\hline GARCH & $\begin{array}{c}2.702 \\
(0.760)\end{array}$ & $\begin{array}{c}0.606 \\
(0.085)\end{array}$ & 29.61 & 0.188 \\
\hline MS-GARCH & $\begin{array}{c}0.641 \\
(1.021)\end{array}$ & $\begin{array}{c}0.730 \\
(0.105)\end{array}$ & 29.08 & 0.203 \\
\hline \multicolumn{5}{|c|}{ Canadian Dollar / US Dollar } \\
\hline Binomial Multifractal & $\begin{array}{l}-0.038 \\
(0.385)\end{array}$ & $\begin{array}{l}1.179 \\
(0.221)\end{array}$ & 1.6339 & 0.217 \\
\hline GARCH & $\begin{array}{c}0.676 \\
(0.243)\end{array}$ & $\begin{array}{c}0.707 \\
(0.121)\end{array}$ & 1.6615 & 0.204 \\
\hline MS-GARCH & $\begin{array}{c}0.630 \\
(0.270)\end{array}$ & $\begin{array}{c}0.754 \\
(0.140)\end{array}$ & 1.6719 & 0.199 \\
\hline
\end{tabular}

Notes: This table gives out of sample forecasting results for the three models. The first two columns correspond to parameter estimates from the Mincer-Zarnowitz OLS regression $e_{t+1}^{2}=\gamma_{0}+\gamma_{1} E_{t}\left(e_{t+1}^{2}\right)+u_{t}$. For an unbiased forecast we expect $\gamma_{0}=0$ and $\gamma_{1}=1$. Asymptotic standard errors in parenthesis are corrected for heteroskedasticity and autocorrelation using the method of Newey and West $(1987,1994)$ and for parameter uncertainty using the method of West and McCracken (1998). MSE is the mean square forecast error, and $R^{2}$ is one less the MSE divided by the sum of squared demeaned squared returns in the out of sample period. 
TABLE 9. - ForECAST SUMMARY, Multiple Horizons

\begin{tabular}{|c|c|c|c|c|c|}
\hline & \multicolumn{5}{|c|}{ "Horizon (Days) } \\
\hline & 1 & 5 & 10 & 20 & 50 \\
\hline \multicolumn{6}{|c|}{ A. Restricted $R^{2}$} \\
\hline \multicolumn{6}{|c|}{ Deutsche Mark / US Dollar } \\
\hline Binomial Multifractal & 0.041 & 0.124 & 0.160 & 0.135 & 0.038 \\
\hline GARCH & 0.035 & 0.069 & 0.033 & -0.147 & -0.761 \\
\hline MS-GARCH & 0.039 & 0.072 & 0.030 & -0.180 & -1.137 \\
\hline \multicolumn{6}{|c|}{ Japanese Yen / US Dollar } \\
\hline Binomial Multifractal & 0.053 & 0.113 & 0.142 & 0.205 & 0.213 \\
\hline GARCH & 0.048 & 0.054 & 0.011 & -0.024 & -0.358 \\
\hline MS-GARCH & 0.048 & 0.044 & -0.009 & -0.067 & -0.569 \\
\hline \multicolumn{6}{|c|}{ British Pound / US Dollar } \\
\hline Binomial Multifractal & 0.057 & 0.165 & 0.235 & 0.250 & 0.273 \\
\hline GARCH & 0.076 & 0.191 & 0.244 & 0.188 & -0.026 \\
\hline MS-GARCH & 0.072 & 0.165 & 0.238 & 0.203 & 0.038 \\
\hline \multicolumn{6}{|c|}{ Canadian Dollar / US Dollar } \\
\hline Binomial Multifractal & 0.051 & 0.172 & 0.221 & 0.217 & 0.111 \\
\hline GARCH & 0.042 & 0.154 & 0.205 & 0.204 & 0.070 \\
\hline MS-GARCH & 0.055 & 0.181 & 0.229 & 0.199 & 0.036 \\
\hline \multicolumn{6}{|c|}{ B. MSE Test vs. Multifractal ( $p$-value) } \\
\hline \multicolumn{6}{|c|}{ Deutsche Mark / US Dollar } \\
\hline GARCH & 0.307 & 0.040 & 0.009 & 0.001 & 0.000 \\
\hline MS-GARCH & 0.314 & 0.004 & 0.000 & 0.000 & 0.000 \\
\hline \multicolumn{6}{|c|}{ Japanese Yen / US Dollar } \\
\hline GARCH & 0.426 & 0.208 & 0.144 & 0.117 & 0.063 \\
\hline MS-GARCH & 0.415 & 0.143 & 0.071 & 0.021 & 0.000 \\
\hline \multicolumn{6}{|c|}{ British Pound / US Dollar } \\
\hline GARCH & 0.906 & 0.824 & 0.606 & 0.156 & 0.016 \\
\hline MS-GARCH & 0.857 & 0.499 & 0.547 & 0.108 & 0.000 \\
\hline \multicolumn{6}{|c|}{ Canadian Dollar / US Dollar } \\
\hline GARCH & 0.294 & 0.3590 & 0.410 & 0.447 & 0.292 \\
\hline MS-GARCH & 0.597 & 0.603 & 0.565 & 0.380 & 0.065 \\
\hline
\end{tabular}

Notes: This table summarizes out of sample forecasting results across multiple horizons. Panel A gives the restricted forecasting $R^{2}$ for each model and horizon. Panel B gives $p$-values from testing that the corresponding model has a lower out of sample forecasting MSE than the binomial multifractal. The tests are corrected for autocorrelation and heteroskedasticity using Newey and West $(1987,1994)$. A low $p$-value indicates that forecasts from the corresponding model would be rejected in favor of multifractal forecasts. 


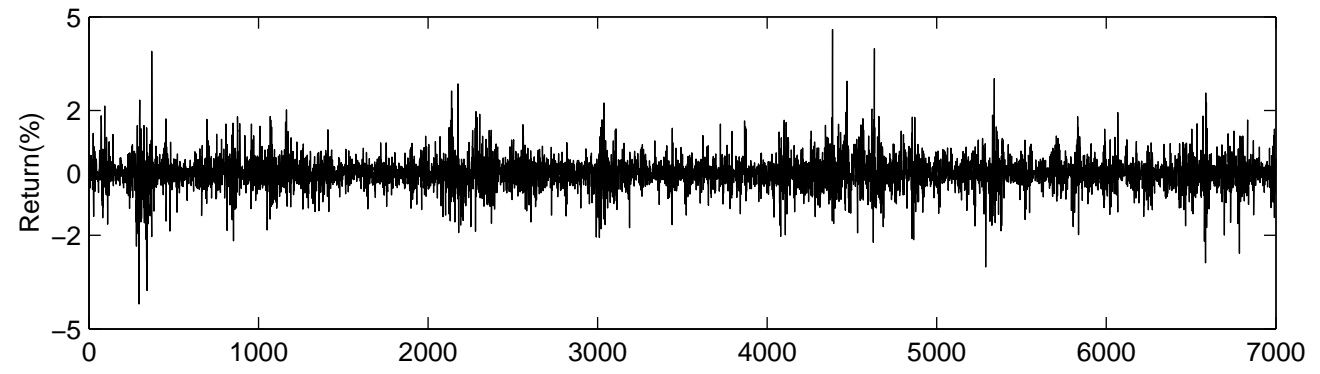

Figure 1: Simulated Multifractal Process. This figure shows simulated log price differences of a multifractal process. The process has $\bar{k}=8$ frequencies and parameter values $m=1.4, \sigma=0.5, \gamma_{\bar{k}}=0.95$, and $b=3$. These parameter values are roughly consistent with estimates that are found to provide a good description of several exchange rate series in later sections of the paper. 
Deutsche Mark (DEM/USD)

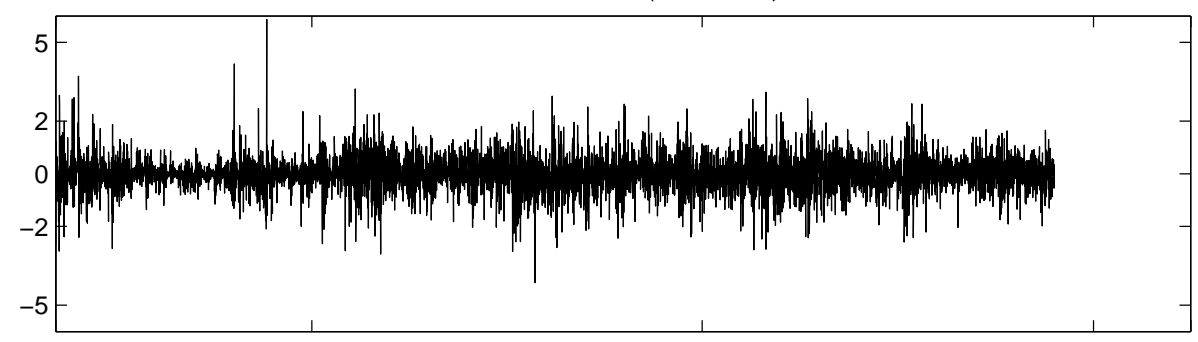

Japanese Yen (JPY/USD)

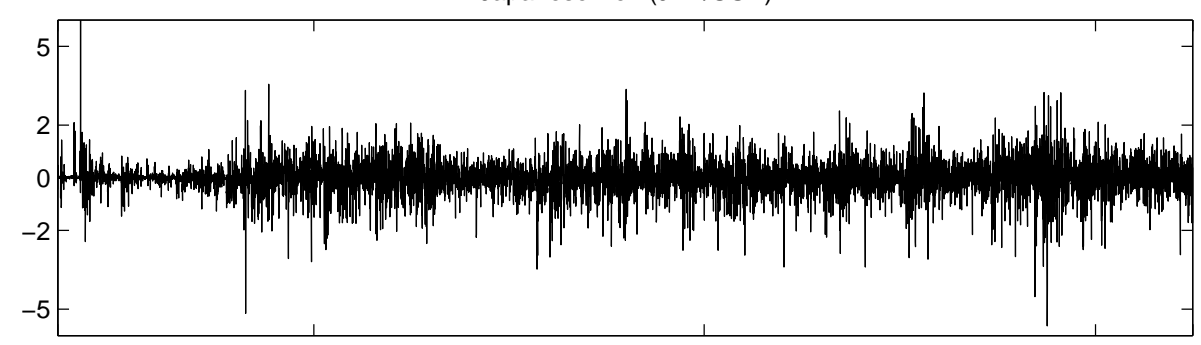

British Pound (GBP/USD)
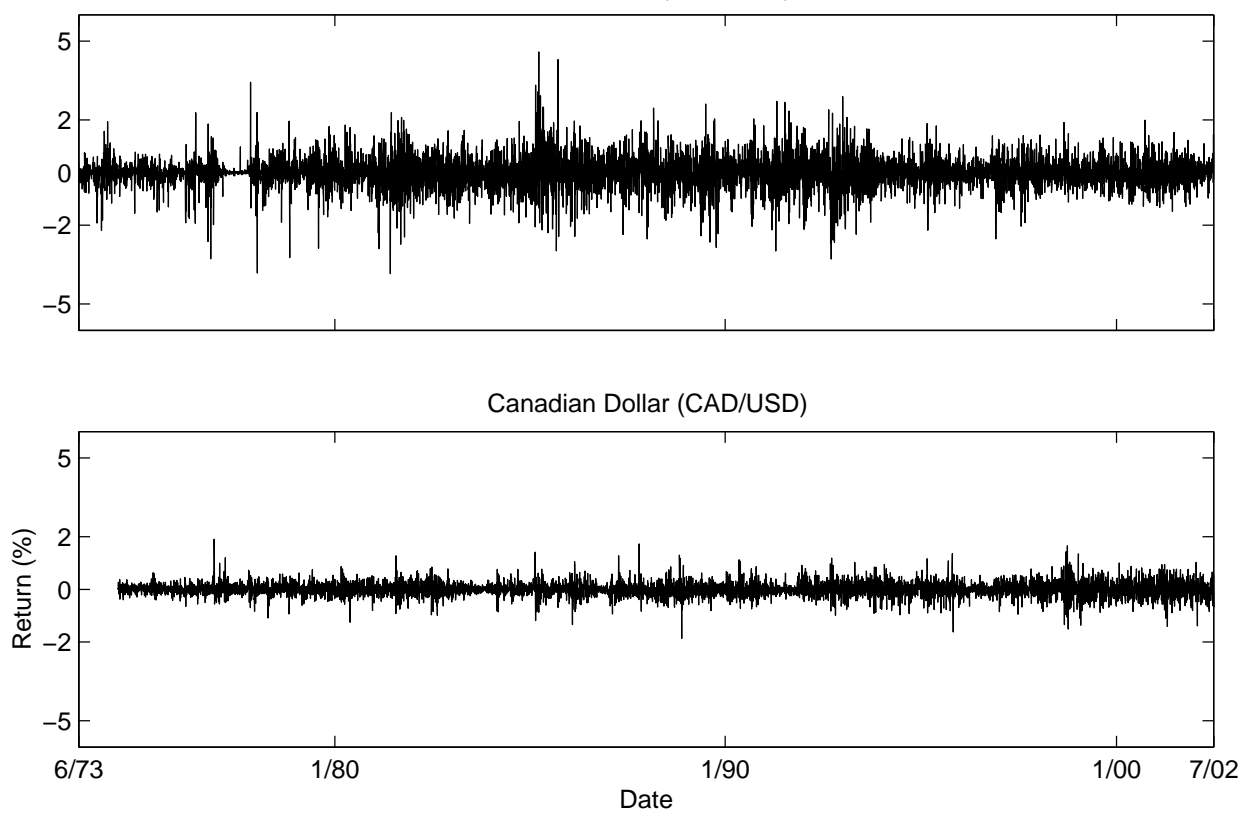

Figure 2: Exchange Rate Data. This figure shows the daily log price differences of the four exchange rate series. 